JOURNAL OF THE AMERICAN MATHEMATICAL SOCIETY

Volume 19, Number 1, Pages 91-118

S 0894-0347(05)00501-1

Article electronically published on September 7, 2005

\title{
ZETA FUNCTION OF REPRESENTATIONS OF COMPACT $p$-ADIC ANALYTIC GROUPS
}

\author{
A. JAIKIN-ZAPIRAIN
}

\section{INTRODUCTION}

Let $G$ be a profinite group. We denote by $r_{n}(G)$ the number of isomorphism classes of irreducible $n$-dimensional complex continuous representations of $G$ (so that the kernel is open in $G$ ). Following [20], we call $r_{n}(G)$ the representation growth function of $G$. If $G$ is a finitely generated profinite group, then $r_{n}(G)<\infty$ for every $n$ if and only if $G$ has the property FAb (that is, $H /[H, H]$ is finite for every open subgroup $H$ of $G$ ) [1, Proposition 2]. In the case when $G$ is a finitely generated pro- $p$ group, the property FAb is equivalent to the condition that all derived subgroups $G^{(k)}$ are open.

In this paper we shall investigate the function

$$
\zeta^{G}(s)=\sum_{n=1}^{\infty} r_{n}(G) n^{-s}=\sum_{\lambda \in \operatorname{Irr}(G)} \lambda(1)^{-s},
$$

when $G$ is an FAb compact $p$-adic analytic group. This function is called the zeta function of representations of $G$. The main result of this article is as follows.

Theorem 1.1. Let $G$ be an FAb compact $p$-adic analytic group with $p>2$. Then there are natural numbers $n_{1}, \ldots, n_{k}$ and functions $f_{1}\left(p^{-s}\right), \ldots, f_{k}\left(p^{-s}\right)$ rational in $p^{-s}$ such that

$$
\zeta^{G}(s)=\sum_{i=1}^{k} n_{i}^{-s} f_{i}\left(p^{-s}\right) .
$$

In particular, if $G$ is an FAb p-adic analytic pro-p group, then $\zeta^{G}(s)$ is a rational function in $p^{-s}$.

The proof of this theorem is based on the correspondence between the characters of a uniform pro- $p$ group and the orbits of the action of the group on the dual of its Lie algebra. This correspondence is an analogue of the Kirillov theory, introduced first in the context of nilpotent Lie groups and then used in many other situations (see 16]). The correspondence is quite explicit, and it also gives the exact formula for characters in some cases. We believe that Theorem 1.1 also holds when $p=2$, but now we only can prove it when $G$ is a uniform pro-2 group (see Theorem 1.2).

Received by the editors June 2, 2004.

2000 Mathematics Subject Classification. Primary 20E18; Secondary 20C15, 20G25, 22E35.

Key words and phrases. Profinite groups, zeta functions, representations.

This work has been supported by the FEDER, MEC Grant MTM2004-04665, and the Ramón y Cajal Program. 
Recall that a pro- $p$ group $G$ is called powerful if $[G, G] \leq G^{p}$ when $p>2$ or $[G, G] \leq G^{4}$ when $p=2$. We say that a pro- $p$ group $G$ is uniform if $G$ is finitely generated, powerful, and without torsion.

Theorem 1.2. Let $N$ be an FAb uniform pro-p group. Then for any $g \in N$,

$$
\sum_{\lambda \in \operatorname{Irr}(G)} \frac{\lambda(g)}{\lambda(1)^{s}}
$$

is a rational function in $p^{-s}$.

The layout of the paper is as follows. In Section 2 we describe Howe's version of Kirillov's correspondence, emphasizing the case $p=2$, which was not considered by Howe. The correspondence permits us to "linearize" the problem and apply the potent tool of $p$-adic integration which we introduce in Section 3 , In Section 4 we prove Theorem 1.2 and deduce Theorem 1.1 when $G$ is uniform. Section 5 is dedicated to some results from the character theory of finite groups. We apply these results in Section 6, where we prove Theorem 1.1. In Section 7 we calculate $\zeta^{\mathbb{S L}_{2}(R)}(s)$, where $R$ is a complete discrete valuation ring with finite residue field of odd characteristic.

The notation is standard. If $G$ is a profinite group, then $\operatorname{Irr}(G)$ denotes the set of the (complex) irreducible smooth (not only linear) characters of $G$. If $L$ is a $\mathbb{Z}_{p}$-Lie lattice of finite rank, then $\operatorname{Irr}(L)$ is the set of the irreducible characters of the additive group of $L$ and $L^{*}=\operatorname{Hom}_{\mathbb{Z}_{p}}\left(L, \mathbb{Z}_{p}\right)$. The set $\operatorname{Ch}(G)$ is the set of the finite dimensional smooth characters of $G$. So any element of $\mathrm{Ch}(G)$ is a finite sum of elements from $\operatorname{Irr}(G)$. If $\mu$ is a Haar measure on $G$ such that $\mu(G)=1$, then the scalar product of two square integrable complex functions $f$ and $g$ is

$$
\langle f, g\rangle=\int_{G} f \bar{g} d \mu .
$$

Let $H$ be an open subgroup of $G$. Given a function $\alpha$ of $G$, we denote by $\alpha_{H}$ its restriction to $H$. If $\phi$ is a class function of $H$, then $\phi^{G}$ denotes the induced class function on $G$. If $N$ is a normal open subgroup of $G$ and $\chi \in \operatorname{Irr}(N)$, then

$$
\operatorname{Irr}(G \mid \chi)=\left\{\lambda \in \operatorname{Irr}(G) \mid\left\langle\lambda_{N}, \chi\right\rangle \neq 0\right\}
$$

and $\operatorname{Ch}(G \mid \chi)$ is the set of finite sums of elements from $\operatorname{Irr}(G \mid \chi)$. We will say that characters from $\operatorname{Ch}(G \mid \chi)$ lie over $\chi$. The set $\operatorname{Irr}(G \mid N)$ is the set of irreducible characters $\lambda$ of $G$ such that $N \not \leq$ ker $\lambda$. We will regard the characters of $G / N$ also as characters of $G$. Thus $\operatorname{Irr}(G)=\operatorname{Irr}(G \mid N) \bigsqcup \operatorname{Irr}(G / N)$. We will use [, $]_{L}$ to denote the Lie bracket and $[,]_{G}$ for the group commutator. If $g$ is an element of a group, $o(g)$ will mean the order of $g$. The order of a nonzero complex number is its order in $\mathbb{C}^{*}$. We will write $\left[N,_{k} M\right]_{G}$ for $[\ldots[N, M], \cdots, M]_{G}$, where $M$ appears $k$ times.

\section{Correspondence between characters and COADJoint orbits OF A UNIFORM PRO- $p$ GROUP}

In this section we describe the correspondence between characters of a uniform pro- $p$ group $N$ and coadjoint orbits of the action of $N$ on the dual of the $\mathbb{Z}_{p}$-Lie lattice associated with $N$. Our approach is based on Howe's papers [12, 11]. Our main contribution in this chapter is an extension of some results from 12 to the case $p=2$. The language of [12] is not so convenient for us, because we do not 
relate the groups under consideration with a fixed field. Therefore, we also present proofs of some results which are contained implicitly in [12].

Recall that according to Lazard (see, for example, [7]) there is an equivalence between the category of uniform pro- $p$ groups and the category of uniform $\mathbb{Z}_{p}$-Lie lattices. The uniform $\mathbb{Z}_{p}$-Lie lattice $\mathbf{N}$ corresponding to a uniform pro-p group $N$ has $N$ itself as its underlying set, and the Lie lattice operations are given in terms of the group operations as follows: for all $z \in \mathbb{Z}_{p}$ and all $x, y \in G$ we have

$$
\begin{aligned}
z \cdot x & =x^{z}, \\
g+h & =\lim _{n \rightarrow \infty}\left(g^{p^{n}} h^{p^{n}}\right)^{p^{-n}}, \\
{[g, h]_{L} } & =\lim _{n \rightarrow \infty}\left[g^{p^{n}}, h^{p^{n}}\right]_{G}^{p^{-2 n}} .
\end{aligned}
$$

Conversely, given a uniform $\mathbb{Z}_{p}$-Lie lattice $\mathrm{N}$, the uniform pro- $p$ group $N$ corresponding to $\mathrm{N}$ can be constructed via the Baker-Campbell-Hausdorff formula $H$. Its underlying set is again $\mathrm{N}$, and the group product of $x, y \in \mathrm{N}$ is given by $x y=H(x, y)$.

Recall that the Baker-Campbell-Hausdorff Formula (BCHF) is $H\left(x_{1}, x_{2}\right)=$ $\log \left(e^{x_{1}} e^{x_{2}}\right)$ regarded as a formal power series in two noncommuting variables. Equivalently, this is a formal power series $H\left(x_{1}, x_{2}\right)$ such that

$$
e^{H\left(x_{1}, x_{2}\right)}=e^{x_{1}} e^{x_{2}} .
$$

The homogeneous component of $H\left(x_{1}, x_{2}\right)$ of degree $n$ is denoted by $H_{n}\left(x_{1}, x_{2}\right)$, so that $H\left(x_{1}, x_{2}\right)=\sum_{n=1}^{\infty} H_{n}\left(x_{1}, x_{2}\right)$. The main fact about the BCHF is that $H_{n}\left(x_{1}, x_{2}\right)$ is a Lie word in $x_{1}$ and $x_{2}$ (see [18, Theorem 9.11]). For example, $H_{1}\left(x_{1}, x_{2}\right)=x_{1}+x_{2}$ and $H_{2}\left(x_{1}, x_{2}\right)=x_{1} x_{2}-x_{2} x_{1}=\left[x_{1}, x_{2}\right]_{L}$.

Let $\left\{e_{k}\right\}$ be a $\mathbb{Z}$-basis of the free Lie algebra generated by $x_{1}$ and $x_{2}$, consisting of simple commutators. Then we can express $H_{n}$ as $H_{n}=\sum \lambda_{k, n} e_{k}$, for some $\lambda_{k, n} \in \mathbb{Q}$. We need the following fact about the coefficients $\lambda_{k, n}$ ([2, Proposition II.8.1]):

$$
v_{p}\left(\lambda_{k, n}\right) \geq-(n-1) /(p-1),
$$

where $v_{p}\left(\frac{a}{b} p^{s}\right)=s$ if $(a, p)=1$ and $(b, p)=1$. We also will use the following well-known formula:

$$
x^{-1} y x=H(H(-x, y), x)=y+\sum_{i=1}^{\infty} \frac{1}{i !}\left[y,{ }_{i} x\right]_{L} .
$$

Although a uniform pro- $p$ group and its associated $\mathbb{Z}_{p}$-Lie lattice are the same as sets, sometimes it will be important to emphasize whether we speak about the group or the lattice. Therefore, in the following if $A, B, N$, etc., are pro- $p$ groups, the associated $\mathbb{Z}_{p}$-Lie lattice will be denoted by $\mathrm{A}, \mathrm{B}, \mathrm{N}$, etc. For example, $\operatorname{Irr}(N)$ will be the set of irreducible characters of the group $N$ and $\operatorname{Irr}(\mathrm{N})$ the irreducible characters of the abelian group $(\mathrm{N},+)$.

The Lazard correspondence can be used not only in the context of uniform pro$p$ groups. Indeed, Lazard [19] constructed his correspondence for a more general family of pro- $p$ groups, saturable pro- $p$ groups. We will call a pro- $p$ group $M$ strong if

(1) $M^{p^{k}}=\left\{m^{p^{k}} \mid m \in M\right\}$,

(2) the map $x \rightarrow x^{p}$ is a bijection between $M$ and $M^{p}$, 
(3) $\left[M^{p^{k}}, M^{p^{k}}\right]_{G} \leq M^{p^{2 k}}$,

(4) operations (2.1) are well defined and $M$ is a $\mathbb{Z}_{p}$-Lie lattice with respect to these operations.

For example, in [13] it is shown that any subgroup of a uniform pro- $p$ group of rank less than $p$ is strong. More results in this direction can be found in 17. It follows implicitly from [10] that any normal subgroup of a uniform pro- $p$ group is strong if $p>2$. In Lemma 2.1 we present a family of strong pro- $p$ groups which appear naturally in this work.

We say that a pro- $p$ group $G$ is $k$-powerful if $[G, G] \leq G^{p^{k}}$. A pro- $p$ group $G$ is $k$-uniform if $G$ is finitely generated, $k$-powerful, and without torsion. Thus, a uniform pro- $p$ group is 1-uniform if $p>2$ and 2-uniform if $p=2$. A pro- $p$ group $H$ is called $k \frac{1}{2}$-uniform if there exists a $(k+1)$-uniform pro- $p$ group $G$ such that $G^{p} \leq H \leq G$. The notions of $k$-uniform and $k \frac{1}{2}$-uniform $\mathbb{Z}_{p}$-Lie lattices are defined in the same way.

Lemma 2.1. Let $A$ be a $k \frac{1}{2}$-uniform pro-p group, where $k=0$ if $p>2$ and $k=1$ if $p=2$. Then the following properties hold:

(1) $A$ is strong, and $\mathrm{A}$ is $k \frac{1}{2}$-uniform;

(2) $\left[\mathrm{A},{ }_{i} \mathrm{~A}\right]_{L} \leq p^{(k+1) i-1} \mathrm{~A}$ : in particular $\left[\mathrm{A},{ }_{i+1} \mathrm{~A}\right]_{L} \leq p^{(k+1) i-1}[\mathrm{~A}, \mathrm{~A}]_{L}$;

(3) $a b \equiv a+b+\frac{1}{2}[a, b]_{L}+\frac{1}{12} \tau \bmod [\mathrm{A}, \mathrm{A}]_{L}$, where $\tau \in[\mathrm{A}, \mathrm{A}, \mathrm{A}]_{L}$;

(4) $x[A, A]_{G}=x+[\mathrm{A}, \mathrm{A}]_{L}$ for every $x \in A$.

Proof. By the definition of a $k \frac{1}{2}$-uniform pro- $p$ group, there exists a $(k+1)$-uniform pro- $p$ group $N$ such that $N^{p} \leq A \leq N$.

(1) We divide the proof of the first statement into several steps:

Step 1. If $x, y \in A$, then $\left(x^{p} y^{p}\right)^{1 / p} \in A$.

Note that $x^{p} y^{p} \equiv(x y)^{p} \bmod N^{p^{2}}$. Since $N$ is powerful, $\left\langle x y, N^{p}\right\rangle$ is also powerful (see [18, Lemma 11.7]). Hence $x^{p} y^{p}=z^{p}$ for some $z \in\left\langle x y, N^{p}\right\rangle \leq A$.

Step 2. If $x, y \in A$, then $\left[x^{p}, y^{p}\right]_{G}^{1 / p^{2}} \in A$.

Note that $\left[x^{p}, y^{p}\right]_{G} \equiv[x, y]_{G}^{p^{2}} \bmod N^{p^{3}}$. Since $N$ is powerful, $\left\langle[x, y]_{G}, N^{p^{2}}\right\rangle$ is also powerful ([18, Lemma 11.7]). Hence $\left[x^{p}, y^{p}\right]_{G}=z^{p^{2}}$ for some $z \in\left\langle[x, y]_{G}, N^{p^{2}}\right\rangle$ $\leq A$.

Step 3. If $x, y \in A$ and $n \geq 1$, then $\left(x^{p^{n}} y^{p^{n}}\right)^{1 / p^{n}} \in A$ and $\left[x^{p^{n}}, y^{p^{n}}\right]_{G}^{1 / p^{2 n}} \in A$.

The case $n=1$ is proved in Steps 1 and 2. Since $N^{p^{2}} \leq A^{p} \leq N^{p}$, we have that $A^{p}$ is uniform. Hence $x^{p^{n}} y^{p^{n}}$ is a $p^{n-1}$-power of an element from $A^{p}$, and so by Step $1, x^{p^{n}} y^{p^{n}}$ is a $p^{n}$-power of an element from $A$. Again using that $A^{p}$ is uniform, we obtain that $\left[x^{p^{n}}, y^{p^{n}}\right]_{G}$ is a $p^{2(n-1)}$-power of an element from $\left[A^{p}, A^{p}\right]$. By Step $2,\left[x^{p^{n}}, y^{p^{n}}\right]_{G}$ is a $p^{2 n}$-power of an element from $A$.

Step 4. Final step.

Since $A$ is a subgroup of $N$ and $N$ is a uniform pro- $p$ group, $A$ satisfies the second property in the definition of a strong pro- $p$ group. The first and the third properties follow from Step 3.

Since $N$ is closed under the operations (2.1), the $\operatorname{limits} \lim _{n \rightarrow \infty}\left(x^{p^{n}} y^{p^{n}}\right)^{p^{-n}}$ and $\lim _{n \rightarrow \infty}\left[x^{p^{n}}, y^{p^{n}}\right]_{G}^{p^{-2 n}}$ exist for any $x, y \in N$. By Step 3, these two limits are in $A$ if $x, y \in A$. Hence $A$ is closed under the operations (2.1), and since $\mathrm{N}$ is a $\mathbb{Z}_{p}$-Lie lattice with respect to this operations, $\mathrm{A}$ is a sublattice. Clearly $\mathrm{A}$ is $k \frac{1}{2}$-uniform. 
(2) Since $\mathbf{N}$ is $(k+1)$-uniform,

$$
\left[\mathrm{A},{ }_{i} \mathrm{~A}\right]_{L} \leq p^{(k+1) i} \mathrm{~N} \leq p^{(k+1) i-1} \mathrm{~A} .
$$

(3) This is a consequence of (2.2) and part (2) of this lemma.

(4) Since

$$
A=\bigsqcup_{x \in A} x[A, A]_{G}=\bigsqcup_{x \in A} x+[\mathrm{A}, \mathrm{A}]_{L},
$$

it is enough to show that $x[A, A]_{G} \leq x+[\mathrm{A}, \mathrm{A}]_{L}$. First, using (2.3), (2.2) and parts (2) and (3) of this lemma, we obtain that $[A, A]_{G} \leq[\mathrm{A}, \mathrm{A}]_{L}$. Then, applying again parts (2) and (3) and (2.2), we conclude that $x[A, A]_{G} \leq x+[\mathrm{A}, \mathrm{A}]_{L}$ for any $x \in A$.

If $N$ is a strong pro- $p$ group, then $N$ acts on $\mathrm{N}$ by conjugation (recall that $N$ and $\mathrm{N}$ coincide as sets). The definition (2.1) of the Lie operations on $\mathrm{N}$ implies that the action by conjugation of an element from $N$ is a Lie homomorphism of $\mathrm{N}$. Hence $N$ also acts on $\operatorname{Irr}(\mathrm{N})$ :

$$
w^{x}(a)=w\left(a^{x^{-1}}\right), a \in \mathbf{N}, x \in N, w \in \operatorname{Irr}(\mathbf{N}) .
$$

The group $\operatorname{Irr}(\mathrm{N})$ is a direct limit of $\operatorname{Irr}\left(\mathrm{N} / p^{n} \mathrm{~N}\right)$, whence any $N$-orbit $\operatorname{in} \operatorname{Irr}(\mathrm{N})$ is finite. If $\Omega \subseteq \operatorname{Irr}(\mathrm{N})$, we define $\Phi_{\Omega}: N \rightarrow \mathbb{C}$ by means of

$$
\Phi_{\Omega}(u)=|\Omega|^{-\frac{1}{2}} \sum_{w \in \Omega} w(u) .
$$

Note that if $\Omega$ is an $N$-orbit in $\operatorname{Irr}(\mathrm{N})$, then $\Phi_{\Omega}$ is a class function on $N$. In the following lemma we collect some basic properties of these functions.

Lemma 2.2. Let $N$ be a strong pro-p group.

(1) Let $\Omega_{1}$ and $\Omega_{2}$ be two $N$-orbits in $\operatorname{Irr}(\mathrm{N})$. Then

$$
\left\langle\Phi_{\Omega_{1}}, \Phi_{\Omega_{2}}\right\rangle= \begin{cases}1 & \text { if } \Omega_{1}=\Omega_{2}, \\ 0 & \text { otherwise. }\end{cases}
$$

In particular, two different orbits $\Omega_{1}$ and $\Omega_{2}$ give two different functions $\Phi_{\Omega_{1}}$ and $\Phi_{\Omega_{2}}$.

(2) Let $K$ be a powerfully embedded open subgroup of $N$. Then $\mathrm{K}$ is a Lie ideal of $\mathrm{N}$ and the functions $\left\{\Phi_{\Omega} \mid \Omega\right.$ is an $N$-orbit in $\left.\operatorname{Irr}(\mathrm{N} / \mathrm{K})\right\}$ form an orthonormal basis for class functions on $N / K$.

Proof. (1) Let $v, w \in \operatorname{Irr}(\mathbf{N})$. Since $\langle v, w\rangle=0$ if $v \neq w$ and $\langle v, w\rangle=1$ if $v=w$, we have the statement.

(2) Since $K$ is powerfully embedded, $a+\mathrm{K}=a K$ for every $a \in N$. Thus $\mathrm{N} / \mathrm{K}=N / K$. The number of $N$-orbits $\operatorname{in} \operatorname{Irr}(\mathrm{N} / \mathrm{K})$ coincides with the number of $N$-orbits in $\mathrm{N} / \mathrm{K}=N / K$. Hence it is equal to the dimension of the space of class functions on $N / K$.

If $w \in \operatorname{Irr}(\mathbf{N})$, we define

$$
B_{w}(l, k)=w\left([l, k]_{L}\right), l, k \in \mathrm{N} .
$$

We see that $B_{w}$ is a bilinear form on N. Put

$$
\operatorname{Rad}(w)=\left\{l \in \mathbf{N} \mid B_{w}(l, \mathbf{N})=1\right\} .
$$

We have the following important result. 
Lemma 2.3 ([12, Lemma 1.1]). Let $\mathrm{N}$ be a $k$-uniform $\mathbb{Z}_{p}$-Lie lattice and $w \in \operatorname{Irr}(\mathrm{N})$. Then $\operatorname{Rad}(w)$ is $k$-uniform. Moreover, if $k \geq 1$ when $p>2$ or $k \geq 2$ when $p=2$, then $\operatorname{Rad}(w)$ and $\operatorname{St}_{N}(w)$ coincide as sets. In particular, $\operatorname{St}_{N}(w)$ is a uniform pro-p group.

Let $w \in \operatorname{Irr}(\mathrm{N})$. We say that a subgroup $\mathrm{A}$ of $(\mathrm{N},+)$ polarizes $w$ in $\mathbf{N}$ if $w\left([\mathrm{~A}, \mathrm{~A}]_{L}\right)=1$ and $\mathrm{A}$ is maximal with respect to this property. Note that in this case $\operatorname{Rad}(w) \leq \mathrm{A}$ and $|\mathrm{A}: \operatorname{Rad}(w)|=|\mathrm{N}: \mathrm{A}|$.

The following lemma is a well-known fact. For instance, its proof is contained in the proof of [12, Lemma 1.4].

Lemma 2.4. Let $V$ be a finite dimensional $\mathbb{F}_{p}$-vector space and $\alpha: V \wedge V \rightarrow \mathbb{C}^{*}$ an antisymmetric bilinear form on $V$. Suppose that a pro-p group $G$ is contained in $\operatorname{Isom}(V, \alpha)$. Then there exists a maximal isotropic subspace $U$ of $(V, \alpha)$ which is $G$-invariant.

Lemma 2.5. Let $\mathrm{N}$ be a $(k+1)$-uniform $\mathbb{Z}_{p}$-Lie lattice and $w \in \operatorname{Irr}(\mathrm{N})$. Suppose that a pro-p group $P$ acts on $\mathrm{N}$ and fixes $w$. Then there exist a polarizing $k \frac{1}{2}$-uniform $\mathbb{Z}_{p}$-Lie lattices $\mathrm{A}$ for $w$, which is stable under the action of $P$.

Proof. We adapt the proof of [12, Lemma 1.4] to our case. First, let us assume that $k=0$ and $w\left(p[\mathrm{~N}, \mathrm{~N}]_{L}\right)=1$. Thus, $p \mathrm{~N} \leq \operatorname{Rad}(w)$ and $B_{w}$ can be considered as a bilinear form on $\mathrm{N} / p \mathrm{~N}$. Hence, by Lemma 2.4, there exists $P$-invariant $p \mathrm{~N} \leq \mathrm{A} \leq \mathrm{N}$ which polarizes $w$.

Now let us assume only that $k=0$. We will prove the lemma by induction on $|\mathbf{N}: \operatorname{Rad}(w)|$. The case $p \mathbf{N} \leq \operatorname{Rad}(w)$ is considered in the previous paragraph. Suppose now that $p \mathrm{~N} \not \leq \operatorname{Rad}(w)$. Let $n \geq 1$ be such that

$$
w\left(\left[p^{n+1} \mathrm{~N}, \mathrm{~N}\right]_{L}\right)=1 \text { and } w\left(\left[p^{n} \mathrm{~N}, \mathrm{~N}\right]_{L}\right) \neq 1 .
$$

Define $\mathbf{T}=\left\{t \in \mathrm{N} \mid w\left(\left[t, p^{n} \mathrm{~N}\right]_{L}\right)=1\right\}$. Note that $\mathrm{T}=\operatorname{Rad}\left(w^{p^{n}}\right)$. Hence, by Lemma 2.3, $\mathrm{T}$ is 1-uniform. Now we can apply the induction hypotheses because $|\mathrm{N}: \operatorname{Rad}(w)|>\left|\mathrm{T}: \operatorname{Rad}\left(w_{\mathrm{T}}\right)\right|$ and $\mathrm{T}$ is $P$-invariant. Hence there exists a polarizing $\frac{1}{2}$-uniform $\mathbb{Z}_{p}$-Lie lattice $\mathrm{A}$ for $w_{\mathrm{T}}$ that is stable under the action of $P$. We show that $\mathbf{A}$ also polarizes $w$. If $b \in \mathbf{N}$ satisfies $w\left([b, \mathrm{~A}]_{L}\right)=1$, then $w\left(\left[b, p^{n} \mathrm{~N}\right]_{L}\right)=1$, because $p^{n} \mathrm{~N} \leq \operatorname{Rad}\left(w_{\mathrm{T}}\right) \leq \mathrm{A}$. Thus, $b \in \mathrm{T}$ and so $b \in \mathrm{A}$.

Now we consider the case $k>1$. Let $\mathbf{M}=p^{-k} \mathrm{~N}$. Then $M$ is a 1-uniform $\mathbb{Z}_{p^{-}}$ lattice. Define $v \in \operatorname{Irr}(\mathrm{M})$ by $v\left(p^{-k} n\right)=w(n)^{p^{k}}$. From the previous discussion we know that there exists a $\frac{1}{2}$-uniform $\mathbb{Z}_{p}$-lattice $\mathrm{B} \leq \mathrm{M}$ polarizing $v$. Then $\mathrm{A}=p^{k} \mathrm{~B}$ is a $k \frac{1}{2}$-uniform $\mathbb{Z}_{p}$-lattice polarizing $w$ in $\mathrm{N}$.

Lemma 2.6. Let $\mathrm{A}$ be a strong $\mathbb{Z}_{p}$-Lie lattice and let $w \in \operatorname{Irr}(\mathrm{A})$ be trivial on $[\mathrm{A}, \mathrm{A}]_{L}$. Then the following hold.

(1) If $\mathrm{A}$ is $k \frac{1}{2}$-uniform with $k=0$ if $p>2$ and $k=1$ if $p=2$, then for every $a \in A$ and $b \in[A, A]_{G}, w(a b)=w(a)$. In particular, $w_{A}$ is a class function on $A$.

(2) If $\mathrm{A}$ is $\frac{1}{2}$-uniform and $p \geq 5$, then $w_{A}$ is a linear character of $A$.

(3) If $\mathrm{A}$ is uniform and $p=3$, then $w_{A}$ is a linear character of $A$.

Proof. The first part of the lemma follows from Lemma 2.1(4) and the second and third from Lemma 2.1(3). 
Lemma 2.7. Let $\mathrm{N}$ be a uniform $\mathbb{Z}_{p}$-Lie lattice and $w, v \in \operatorname{Irr}(\mathrm{N})$. Let $\mathrm{A}$ be a polarizing $\mathbb{Z}_{p}$-Lie lattice for $w$ (as in Lemma 2.5). Then $v_{A}=w_{A}$ if and only if $v$ and $w$ are $A$-conjugate.

Proof. Note first that by Lemma 2.6(1), if $v=w^{a}$ for some $a \in A$, then for any $b \in A$,

$$
v(b)=w^{a}(b)=w\left(b^{a^{-1}}\right)=w(b) .
$$

Now let $\Sigma$ be the set of elements $v$ of $\operatorname{Irr}(\mathrm{N})$ such that $v_{A}=w_{A}$. We have that $\Sigma$ has $|\mathrm{N}: \mathrm{A}|$ elements. On the other hand, $A$ acts on $\Sigma$ and the $A$-orbit of $w$ has

$$
\left|A: \operatorname{St}_{N}(w)\right|=|\mathrm{A}: \operatorname{Rad}(w)|=|\mathrm{N}: \mathrm{A}|=|\Sigma|
$$

elements (we have used Lemma 2.3). Hence $\Sigma$ is exactly the $A$-orbit of $w$.

Lemma 2.8. Let $\mathrm{N}$ be a uniform $\mathbb{Z}_{p}$-Lie lattice and $w \in \operatorname{Irr}(\mathrm{N})$. Let $\mathrm{A}$ be a polarizing $\mathbb{Z}_{p}$-Lie lattice for $w$ (as in Lemma 2.5). Suppose that $\Omega$ is the $N$-orbit of $w$ in $\operatorname{Irr}(\mathbf{N})$. Then $\Phi_{\Omega}=\left(w_{A}\right)^{N}$.

Proof. Let $\bar{w}=\frac{1}{|N: A|}\left(1_{A}\right)^{N} w$, i.e., $\bar{w}(g)=0$ if $g \notin A$ and $\bar{w}(g)=w(g)=w_{A}(g)$ if $g \in A$. Fix a transversal $\left\{g_{i}\right\}$ for the right cosets of $A$ in $N$. By the definition of $\left(w_{A}\right)^{N}$, we have that

$$
\left(w_{A}\right)^{N}=\sum_{i} \bar{w}^{g_{i}}
$$

Let $\Sigma$ be the set of elements $v$ of $\operatorname{Irr}(\mathrm{N})$ such that $v_{A}=w_{A}$. Then, by Lemma 2.7 .

$$
\bar{w}=\frac{1}{|N: A|} \sum_{v \in \Sigma} v=\frac{1}{|N: A|} \sum_{j} w^{h_{j}}
$$

where $\left\{h_{j}\right\}$ is a transversal for the right cosets of $\operatorname{St}_{G}(w)$ in $A$. Together with (2.4) this gives us that $\left(w_{A}\right)^{N}=\Phi_{\Omega}$.

2.1. The case $p>2$. Now we consider the case $p>2$.

Theorem 2.9. Let $N$ be a uniform pro-p group, with $p>2$, and $\Omega$ an $N$-orbit in $\operatorname{Irr}(\mathrm{N})$. Then $\Phi_{\Omega} \in \operatorname{Irr}(N)$ and all characters of $N$ have this form.

Proof. Let us first assume that $p \geq 5$. Then by Lemmas 2.8 and 2.6, $\Phi_{\Omega}$ is a class function induced from a linear character of a subgroup. Hence $\Phi_{\Omega}$ is a character of $N$. By Lemma 2.2(1), $\Phi_{\Omega}$ has norm 1, and so $\Phi_{\Omega}$ is an irreducible character. Using Lemma $2.2(2)$, we obtain that $\left\{\Phi_{\Sigma} \mid \Sigma\right.$ is an $N$-orbit in $\left.\operatorname{Irr}(\mathrm{N})\right\}$ is the set of all irreducible characters of $N$.

Now suppose that $p=3$. The argument of the previous paragraph does not work in this case, because not every $w \in \operatorname{Irr}(\mathrm{A})$ that is trivial on $[\mathrm{A}, \mathrm{A}]_{L}$ is a character of $A$ when $A$ is $\frac{1}{2}$-uniform (see Lemma 2.6). We will use another observation. Note that any orthogonal matrix over $\mathbb{Q}$ with nonnegative entries is a permutation matrix. Therefore, if we have two orthonormal bases and the matrix of change of bases has only nonnegative rational entries, then these two bases coincide. Hence it is enough to prove that for any $\lambda \in \operatorname{Irr}(N)$ and any $N$-orbit $\Omega$ in $\operatorname{Irr}(\mathrm{N})$ the scalar product $\left\langle\lambda, \Phi_{\Omega}\right\rangle$ is nonnegative. We divide the proof into several steps:

Step 1. Let $N$ be a uniform pro-3 group, and let $w \in \operatorname{Irr}(\mathrm{N})$ be $N$-invariant. Then $\langle w, \lambda\rangle$ is a nonnegative integer for any $\lambda \in \operatorname{Irr}(N)$. 
By Lemma 2.6, $w$ is a linear character of $N$. Therefore, $\langle w, \lambda\rangle$ is a nonnegative integer for any $\lambda \in \operatorname{Irr}(N)$.

Step 2. Let $N$ be a uniform pro-3 group, and let $w \in \operatorname{Irr}(\mathrm{N})$ be such that $w\left([\mathrm{~N}, \mathrm{~N}, \mathrm{~N}]_{L}\right)=1$. If $\Omega$ is the $N$-orbit of $w$, then $\left\langle\Phi_{\Omega}, \lambda\right\rangle$ is a nonnegative rational number for any $\lambda \in \operatorname{Irr}(N)$.

Since $w\left([\mathbf{N}, \mathbf{N}, \mathbf{N}]_{L}\right)=1, w\left(3^{2-s}\left[\mathbf{N},{ }_{s} \mathbf{N}\right]_{L}\right)=1$. Hence if $a \in \operatorname{St}_{N}(w)$ and $x \in N$, then, by (2.3),

$$
w^{x}(a)=w\left(a^{x^{-1}}\right)=w\left(a+[a,-x]_{L}+\frac{1}{2 !}[a,-x,-x]_{L}+\cdots\right)=w(a) .
$$

Thus if $v \in \Omega, v_{\mathrm{St}_{N}(w)}=w_{\mathrm{St}_{N}(w)}$. Since there are only $\left|N: \operatorname{St}_{N}(w)\right|=|\Omega|$ linear characters of $\mathrm{N}$ satisfying this property, they are all in $\Omega$. Hence $\Phi_{\Omega}(a)=0$ for any $a \notin \operatorname{St}_{N}(w)$. Thus,

$$
\left\langle\Phi_{\Omega}, \lambda\right\rangle=\frac{1}{|\Omega|}\left\langle\left(\Phi_{\Omega}\right)_{\mathrm{St}_{N}(w)}, \lambda_{\mathrm{St}_{N}(w)}\right\rangle=\frac{1}{|\Omega|^{1 / 2}}\left\langle w_{\mathrm{St}_{N}(w)}, \lambda_{\mathrm{St}_{N}(w)}\right\rangle
$$

is a nonnegative rational number by Step 1 .

Step 3. Let $N$ be a uniform pro-3 group. Then for any $\lambda \in \operatorname{Irr}(N)$ and any $N$-orbit $\Omega$ in $\operatorname{Irr}(\mathrm{N})$ the scalar product $\left\langle\lambda, \Phi_{\Omega}\right\rangle$ is a nonnegative rational number.

By Lemma 2.5 there exists a $\frac{1}{2}$-uniform lattice A polarizing $w$ in N. Put

$$
\mathrm{B}=\mathrm{A}+\sum_{i=1}^{\infty} \frac{1}{3^{i}}\left[\mathrm{~A},,_{i} \mathrm{~A}\right]_{L} .
$$

Note that $B$ is uniform. Since $A$ is $\frac{1}{2}$-uniform, $\left[A,{ }_{i+1} A\right]_{L} \leq 3^{i-1}[A, A]_{L}$ by Lemma 2.1(2). Hence

$$
[\mathrm{B}, \mathrm{B}, \mathrm{B}]_{L} \leq \sum_{i=1}^{\infty} \frac{1}{3^{i-1}}\left[\mathrm{~A},_{i+1} \mathrm{~A}\right]_{L} \leq[\mathrm{A}, \mathrm{A}]_{L} .
$$

By Lemma 2.8, $w_{A}^{B}=\Phi_{\Sigma}$, where $\Sigma$ is the $B$-orbit of $w_{\mathrm{B}}$ in $\operatorname{Irr}(\mathrm{B})$. Thus, we obtain that

$$
\left\langle\lambda, \Phi_{\Omega}\right\rangle=\left\langle\lambda, w_{A}^{N}\right\rangle=\left\langle\lambda_{A}, w_{A}\right\rangle=\left\langle\lambda_{B}, w_{A}^{B}\right\rangle=\left\langle\lambda_{B}, \Phi_{\Sigma}\right\rangle
$$

is a nonnegative rational number by Step 2 .

As we have indicated previously, Step 3 implies the theorem in the case $p=3$.

2.2. The case $p=2$. Let $N$ be a uniform pro-2 group. The main difference between the cases $p=2$ and $p>2$ is that not every $N$-invariant $w \in \operatorname{Irr}(\mathrm{N})$ is a linear character of $N$ (see Lemma 2.6). Moreover, $w$ is a linear character of $N$ if and only if $w$ is trivial on $2^{-1}[\mathrm{~N}, \mathrm{~N}]_{L}$.

Let us study first linear characters of a uniform pro- 2 group. Let $B$ be a $1 \frac{1}{2}$ uniform pro-2 group. Put $A=B^{2}$, and let $\mu \in \operatorname{Irr}(A)$ be a linear character of $A$. Let $w \in \operatorname{Irr}(\mathrm{B})$ be such that $\left\langle\mu, w_{A}\right\rangle \neq 0$. Note that $w_{A}$ is $A$-stable.

Lemma 2.10. The functions $w$ and $\mu$ coincide on $\operatorname{St}_{A}\left(w_{\mathrm{B}}\right)$. In particular, $w(a)^{2}=$ $\mu(a)^{2}$ for any $a \in A$. 
Proof. By Lemma 2.3 $\mathrm{St}_{A}\left(w_{B}\right)=\operatorname{Rad}\left(w_{\mathrm{B}}\right) \cap A$, whence if $a \in A$ and $x \in \operatorname{St}_{A}\left(w_{B}\right)$, then $w\left(\left[\frac{1}{2} a, x\right]_{L}\right)=1$. Therefore, using Lemma 2.1(3), we obtain that

$$
w(a x)=w\left(a+x+\frac{1}{2}[a, x]_{L}\right)=w\left(a+x+\left[\frac{1}{2} a, x\right]_{L}\right)=w(a) w(x) .
$$

In particular, we see that $w_{\mathrm{St}_{A}\left(w_{B}\right)} \in \operatorname{Irr}\left(\operatorname{St}_{A}\left(w_{B}\right)\right)$. Let $R$ be a transversal of $A$ over $\operatorname{St}_{A}\left(w_{B}\right)$. Then we have

$$
\left\langle\mu, w_{A}\right\rangle=\frac{1}{|R|} \sum_{r \in R} \mu(r) \overline{w(r)}\left\langle w_{\mathrm{St}_{A}\left(w_{B}\right)}, \mu_{\mathrm{St}_{A}\left(w_{B}\right)}\right\rangle .
$$

Hence $\left\langle w_{\mathrm{St}_{A}\left(w_{B}\right)}, \mu_{\mathrm{St}_{A}\left(w_{B}\right)}\right\rangle \neq 0$, and so $w_{\mathrm{St}_{A}\left(w_{B}\right)}=\mu_{\mathrm{St}_{A}\left(w_{B}\right)}$.

Since $A^{2} \leq \operatorname{St}_{A}\left(w_{B}\right)$,

$$
w(a)^{2}=w\left(a^{2}\right)=\mu\left(a^{2}\right)=\mu(a)^{2}
$$

for any $a \in A$.

For every $a \in B$ and $x \in A$ define $\gamma_{a}(x)=w\left([x, a]_{L}\right)$ and $c(x)=\mu(x) w(x)^{-1}$. By the previous lemma $c(x)= \pm 1$. It is clear that $\gamma_{a}$ is a linear character of $A$ of order 2. Moreover, $\gamma_{a}=1_{A}$ if and only if $a \in \operatorname{St}_{B}\left(w_{A}\right)$.

Lemma 2.11. Let $T$ be a transversal of $B$ over $\operatorname{St}_{B}\left(w_{A}\right)$, and let $x, y \in A$. Then we have the following.

(1) $\operatorname{Irr}\left(A / \operatorname{St}_{A}\left(w_{B}\right)\right)=\left\{\gamma_{a} \mid a \in T\right\}$.

(2) $c(x y)=c(x) c(y) w\left(\frac{[x, y]_{L}}{2}\right)$.

(3) $c(x)=\frac{\operatorname{sign}\left(\left\langle\mu, w_{A}\right\rangle\right)}{|T|^{1 / 2}} \sum_{a \in T} c\left(a^{2}\right) \gamma_{a}$.

(4) $\mu(x)=\frac{\operatorname{sign}\left(\left\langle\mu, w_{A}\right\rangle\right)}{|T|^{1 / 2}} \sum_{a \in T} c\left(a^{2}\right) w^{a}(x)$. In particular, if $v \in \operatorname{Irr}(\mathbf{A})$, then $\langle\mu, v\rangle \neq 0$ if and only if $v=w^{a}$ for some $a \in B$.

Proof. (1) By Lemma 2.3. $\operatorname{St}_{A}\left(w_{B}\right)=\operatorname{Rad}(w) \cap A$, whence $\gamma_{a}(x)=1$ for any $x \in$ $\operatorname{St}_{A}\left(w_{B}\right)$. Thus, we obtain that $\left\{\gamma_{a} \mid a \in T\right\} \subseteq \operatorname{Irr}\left(A / \operatorname{St}_{A}\left(w_{B}\right)\right)$. Since all $\gamma_{a}(a \in$ $T)$ are different and $|T|=\left|A / \operatorname{St}_{A}\left(w_{B}\right)\right|$, we have the equality $\operatorname{Irr}\left(A / \operatorname{St}_{A}\left(w_{B}\right)\right)=$ $\left\{\gamma_{a} \mid a \in T\right\}$.

(2) By Lemma 2.1(3), we have

$$
\begin{aligned}
c(x y) & =\mu(x y) w(x y)^{-1}=\mu(x) \mu(y) w\left(x+y+\frac{[x, y]_{L}}{2}\right) \\
& =\mu(x) \mu(y) w(x) w(y) w\left(\frac{[x, y]_{L}}{2}\right)=c(x) c(y) w\left(\frac{[x, y]_{L}}{2}\right) .
\end{aligned}
$$

(3) By Lemma 2.10 and the previous item, $c$ can be considered as a function on $A / \operatorname{St}_{A}\left(w_{B}\right)$. Since $\operatorname{Irr}\left(A / \operatorname{St}_{A}\left(w_{B}\right)\right)=\left\{\gamma_{a} \mid a \in T\right\}$, there are $c_{a}(a \in T)$ such that $c=\sum_{a \in T} c_{a} \gamma_{a}$. If $a \in A$, then

$$
\begin{aligned}
c_{a} & =\left\langle c, \gamma_{a}\right\rangle=\int_{A} c(x) \gamma_{a}(x) d x=\int_{A} c(x) w\left(\left[a^{2}, x\right] / 2\right) d x \\
& =\int_{A} c\left(x a^{2}\right) c\left(a^{2}\right) d x=c\left(a^{2}\right)\left\langle w_{A}, \mu\right\rangle .
\end{aligned}
$$

Since $1=\langle c, c\rangle=\sum_{a \in T} c_{a}^{2}=|T|\left\langle w_{A}, \mu\right\rangle^{2}$, we obtain that $\left\langle w_{A}, \mu\right\rangle^{2}=1 /|T|$. Thus, we conclude that

$$
c_{a}=\frac{\operatorname{sign}\left(\left\langle\mu, w_{A}\right\rangle\right) c\left(a^{2}\right)}{|T|^{1 / 2}} .
$$

(4) Since $\mu(x)=w(x) c(x)$ and $w^{a}(x)=w(x) \gamma_{a}(x)$, we obtain the desired result. 
Now we are ready to describe the characters of an arbitrary uniform pro-2 group.

Theorem 2.12. Let $N$ be a uniform pro-2 group. Then there exists a bijection $f$ between $\operatorname{Irr}(N)$ and $\left\{\Phi_{\Delta} \mid \Delta\right.$ is an $N$-orbit in $\left.\operatorname{Irr}(\mathrm{N})\right\}$ such that for any $\phi \in$ $\operatorname{Irr}(N), f(\phi)_{N^{2}}=\phi_{N^{2}}$. Moreover, if $N$ is 3-uniform, then there exists a function $d_{\phi}: N / N^{2} \rightarrow \pm 1$ with $f(\phi)(a)=d_{\phi}(a) \phi(a)$ for any $a \in N$.

Proof. Let $\lambda \in \operatorname{Irr}\left(N^{2}\right)$, and take $\phi \in \operatorname{Irr}(N)$ lying over $\lambda$. Choose $w \in \operatorname{Irr}(\mathrm{N})$ such that $\left\langle w_{N^{2}}, \lambda\right\rangle \neq 0$. Let $\mathrm{B}$ be a $1 \frac{1}{2}$-uniform $\mathbb{Z}_{p}$-Lie lattice polarizing $w^{4}$ in $\mathbf{N}$ (see Lemma 2.5). Then $\mathrm{A}=2 \mathrm{~B}$ is a $2 \frac{1}{2}$-uniform lattice polarizing $w_{2 \mathrm{~N}}$ in $2 \mathrm{~N}$. We will divide the proof of the theorem into several steps:

Step 1. There exists a linear character $\mu \in \operatorname{Irr}(A)$ such that $\mu$ is a constituent of $\lambda_{A}$ and $\left\langle\mu, w_{A}\right\rangle \neq 0$.

Let $\Omega$ be the $N^{2}$-orbit of $w_{2 \mathrm{~N}}$ in $\operatorname{Irr}(2 \mathrm{~N})$. By Lemma 2.8 ,

$$
\left\langle\left(w_{A}\right)^{N^{2}}, \lambda\right\rangle=\left\langle\Phi_{\Omega}, \lambda\right\rangle=\left|N^{2}: A\right|\left\langle w_{N^{2}}, \lambda\right\rangle \neq 0 .
$$

Hence, by Frobenius reciprocity,

$$
\left\langle w_{A}, \lambda_{A}\right\rangle=\left\langle\left(w_{A}\right)^{N^{2}}, \lambda\right\rangle \neq 0
$$

Thus, there exists an irreducible constituent $\mu \in \operatorname{Irr}(A)$ of $\lambda_{A}$ such that $\left\langle\mu, w_{A}\right\rangle \neq 0$. By Lemma 2.6(1), $\mu$ is linear.

From now on we fix one such linear character $\mu \in \operatorname{Irr}(A)$.

Step 2. We have $\operatorname{St}_{B}(\mu)=\operatorname{St}_{B}\left(w_{A}\right)=\operatorname{St}_{N}\left(w_{N^{2}}\right) A$. In particular, $\operatorname{St}_{B}\left(w_{A}\right) \cap$ $N^{2}=A$.

The equality $\operatorname{St}_{B}(\mu)=\operatorname{St}_{B}\left(w_{A}\right)$ follows from Lemma 2.11(4). Also $\operatorname{St}_{N}\left(w_{N}\right) A$ is clearly contained in $\operatorname{St}_{B}\left(w_{A}\right)$. Now let $x \in \mathrm{St}_{B}\left(w_{A}\right)$. Then by Lemma2.7 $w^{x}=w^{a}$ for some $a \in A$. Hence $x a^{-1} \in \operatorname{St}_{N}\left(w_{N^{2}}\right)$, and so $x \in \operatorname{St}_{N}\left(w_{N^{2}}\right) A$.

Step 3. We have $\lambda=\mu^{N^{2}}$.

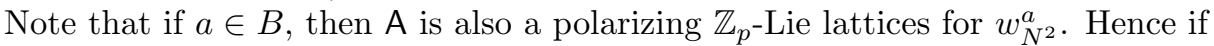
$a, b \in B$, then, by Lemma 2.8, $\left(w_{A}^{a}\right)^{N^{2}}=\left(w_{A}^{b}\right)^{N^{2}}$ if and only if

$$
a b^{-1} \in\left(\operatorname{St}_{N}\left(w_{N^{2}}\right) N^{2}\right) \cap B=\operatorname{St}_{N}\left(w_{N^{2}}\right)\left(N^{2} \cap B\right)=\operatorname{St}_{B}\left(w_{A}\right)\left(N^{2} \cap B\right) .
$$

Let $T_{1}$ be a transversal of $N^{2} \cap B$ over $A$, and let $T_{2}$ be a transversal of $B$ over $\mathrm{St}_{B}\left(w_{A}\right)\left(N^{2} \cap B\right)$. By Step $2, \mathrm{St}_{B}\left(w_{A}\right) \cap N^{2} \leq A$. Hence $T_{1} T_{2}$ is a transversal of $B$ over $\operatorname{St}_{B}\left(w_{A}\right)$. Put $T=T_{1} T_{2}$.

Using Lemma 2.11(4), we obtain that

$$
\mu(x)=\frac{\operatorname{sign}\left(\left\langle\mu, w_{A}\right\rangle\right)}{|T|^{1 / 2}} \sum_{a \in T} c\left(a^{2}\right) w_{A}^{a}(x),
$$

where $c(x)=\mu(x) w(x)^{-1}$ and $x \in A$. In order to prove that $\lambda=\mu^{N^{2}}$, we have to 
show that $\left\langle\mu^{N^{2}}, \mu^{N^{2}}\right\rangle=1$ :

$$
\begin{aligned}
\left\langle\mu^{N^{2}}, \mu^{N^{2}}\right\rangle & =\left\langle\frac{\operatorname{sign}\left(\left\langle\mu, w_{A}\right\rangle\right)}{|T|^{1 / 2}} \sum_{a \in T} c\left(a^{2}\right)\left(w_{A}^{a}\right)^{N^{2}}, \frac{\operatorname{sign}\left(\left\langle\mu, w_{A}\right\rangle\right)}{|T|^{1 / 2}} \sum_{a \in T} c\left(a^{2}\right)\left(w_{A}^{a}\right)^{N^{2}}\right\rangle \\
& =\frac{1}{|T|} \sum_{a_{2} \in T_{2}} \sum_{a_{1}, b_{1} \in T_{1}} c\left(a_{1}^{2} a_{2}^{2}\right) c\left(b_{1}^{2} a_{2}^{2}\right) \text { (we use (2.5)) } \\
& =\frac{1}{|T|\left|T_{1}\right|} \sum_{a \in T} \sum_{a_{1}, b_{1} \in T_{1}} c\left(a_{1}^{2} a^{2}\right) c\left(b_{1}^{2} a^{2}\right) \\
& =\frac{1}{|T|\left|T_{1}\right|} \sum_{a_{1}, b_{1} \in T_{1}}\left(\sum_{a \in T} c\left(a_{1}^{2}\right) c\left(b_{1}^{2}\right) \gamma_{a_{1}}\left(a^{2}\right) \gamma_{b_{1}}\left(a^{2}\right)\right) \\
& =\frac{1}{\left|T_{1}\right|} \sum_{a_{1}, b_{1} \in T_{1}} c\left(a_{1}^{2}\right) c\left(b_{1}^{2}\right)\left\langle\gamma_{a_{1}}, \gamma_{b_{1}}\right\rangle \\
& =\frac{1}{\left|T_{1}\right|} \sum_{a_{1} \in T_{1}} c\left(a_{1}^{2}\right) c\left(a_{1}^{2}\right)=1 .
\end{aligned}
$$

Step 4. We have $\operatorname{St}_{N}(\lambda)=\operatorname{St}_{N}\left(w_{N^{2}}\right) N^{2}$.

Let $\Sigma$ be the $B N^{2}$-orbit of $w_{2 N}$ in $\operatorname{Irr}(2 N)$. Then, using the previous step and the expression for $\mu$ from Lemma 2.11 (4), we obtain that there are $c_{v} \in \mathbb{Q}(v \in \Sigma)$ such that

$$
\lambda=\sum_{v \in \Sigma} c_{v} v
$$

By Step $2, \operatorname{St}_{N}\left(w_{N^{2}}\right) \leq \operatorname{St}_{B}(\mu)$, whence $\operatorname{St}_{N}\left(w_{N^{2}}\right) N^{2} \leq \operatorname{St}_{N}(\lambda)$.

Now, suppose $x \in \operatorname{St}_{N}(\lambda)$. Using (2.6), we obtain that $x \in B N^{2}$. Hence we only need to consider the case $x \in B$.

Let $\alpha=\sum_{t \in T} w_{A}^{t}$. Then, by (2.5),$\left\langle\alpha^{N^{2}}, \alpha^{N^{2}}\right\rangle=\left|T_{2}\right|\left|T_{1}\right|^{2}$. On the other hand, by Lemmas 2.11(1) and 2.10.

$$
\alpha=\sum_{t \in T} w_{A}^{t}=1_{\mathrm{St}_{A}\left(w_{B}\right)}^{A} w_{A}=1_{\mathrm{St}_{A}\left(w_{B}\right)}^{A} \mu=\sum_{t \in T} \mu^{t} .
$$

Hence $\left\langle\alpha^{N^{2}}, \alpha^{N^{2}}\right\rangle=\left|B: \operatorname{St}_{B}(\lambda)\right|\left|\operatorname{St}_{B}(\lambda): \operatorname{St}_{B}\left(w_{A}\right)\right|^{2}$. Since $\operatorname{St}_{B}\left(w_{A}\right)\left(B \cap N^{2}\right) \leq$ $\mathrm{St}_{B}(\lambda)$, we conclude that $\operatorname{St}_{B}\left(w_{A}\right)\left(B \cap N^{2}\right)=\operatorname{St}_{B}(\lambda)$.

Step 5. The map $\beta \mapsto \beta^{\operatorname{St}_{N}(\lambda)}$ is a bijection between $\operatorname{Irr}\left(\operatorname{St}_{B}(\mu) \mid \mu\right)$ and $\operatorname{Irr}\left(\operatorname{St}_{N}(\lambda) \mid \lambda\right)$.

Since $\mu^{N^{2}}=\lambda$ and $\operatorname{St}_{N}(\lambda)$ fixes $\lambda, \beta^{\operatorname{St}_{N}(\lambda)}$ lies over $\lambda$ for every $\beta \in \operatorname{Irr}\left(\operatorname{St}_{B}(\mu) \mid \mu\right)$. In particular, we have that

$$
\left\langle\mu^{\mathrm{St}_{N}(\lambda)}, \mu^{\mathrm{St}_{N}(\lambda)}\right\rangle=\left\langle\lambda^{\mathrm{St}_{N}(\lambda)}, \lambda^{\mathrm{St}_{N}(\lambda)}\right\rangle=\left\langle\lambda,\left(\lambda^{\mathrm{St}_{N}(\lambda)}\right)_{N^{2}}\right\rangle=\left|\mathrm{St}_{N}(\lambda) / N^{2}\right| .
$$

On the other hand,

$$
\begin{aligned}
\mu^{\mathrm{St}_{N}(\lambda)} & =\left(\mu^{\mathrm{St}_{B}(\mu)}\right)^{\mathrm{St}_{N}(\lambda)} \\
& =\left(\sum_{\gamma \in \operatorname{Irr}\left(\mathrm{St}_{B}(\mu) \mid \mu\right)} \gamma(1) \gamma\right)^{\mathrm{St}_{N}(\lambda)}=\sum_{\gamma \in \operatorname{Irr}\left(\operatorname{St}_{B}(\mu) \mid \mu\right)} \gamma(1) \gamma^{\mathrm{St}_{N}(\lambda)} .
\end{aligned}
$$


Hence we have

$$
\begin{aligned}
\left|\mathrm{St}_{N}(\lambda) / N^{2}\right| & =\left\langle\mu^{\mathrm{St}_{N}(\lambda)}, \mu^{\mathrm{St}_{N}(\lambda)}\right\rangle=\sum_{\gamma, \delta \in \operatorname{Irr}\left(\mathrm{St}_{B}(\mu) \mid \mu\right)} \gamma(1) \delta(1)\left\langle\gamma^{\mathrm{St}_{N}(\lambda)}, \delta^{\mathrm{St}_{N}(\lambda)}\right\rangle \\
& \leq \sum_{\gamma \in \operatorname{Irr}\left(\mathrm{St}_{B}(\mu) \mid \mu\right)} \gamma(1)^{2}=\left|\mathrm{St}_{B}(\mu) / A\right|=\left|\mathrm{St}_{N}(\lambda) / N^{2}\right| .
\end{aligned}
$$

The last equality is a consequence of Step 2 and Step 4. Thus, we obtain that if $\gamma, \delta \in \operatorname{Irr}\left(\operatorname{St}_{B}(\mu) \mid \mu\right)$, then

$$
\left\langle\gamma^{\mathrm{St}_{N}(\lambda)}, \delta^{\mathrm{St}_{N}(\lambda)}\right\rangle=\left\{\begin{array}{lll}
1 & \text { if } & \gamma=\delta \\
0 & \text { if } & \gamma \neq \delta
\end{array}\right.
$$

which proves Step 5 .

Let $\psi$ be a character of $\operatorname{St}_{N}(\lambda)$ such that $\phi=\psi^{N}$. By Step 5 , there exists a character $\beta \in \operatorname{Irr}\left(\operatorname{St}_{B}(\mu) \mid \mu\right)$ such that $\psi=\beta^{\operatorname{St}_{N}(\lambda)}$. Put $\gamma=\beta^{B}$. Hence $\gamma^{N}=$ $\beta^{N}=\psi^{N}=\phi$. Let $C$ be a subgroup of $\operatorname{St}_{B}(\mu)$ such that $\beta=\delta^{\operatorname{St}_{B}(\mu)}$ and $\delta$ is a linear character of $C$. Since $A$ is contained in the center of $\beta, A \leq C$ and so $\delta$ is an extension of $\mu$. Note that since $[C, C]_{G} \in A^{2}$,

$$
w\left([C, C]_{G}\right)=\mu\left([C, C]_{G}\right)=\delta\left([C, C]_{G}\right)=1 .
$$

Step 6. The subgroup $C$ is $1 \frac{1}{2}$-uniform, and $C$ is a polarizing lattice for $w$ in N. Put $\mathrm{D}=\operatorname{Rad}\left(w_{\mathrm{N}}^{2}\right)+\mathrm{A}$. By Lemma 2.3 .

$$
\operatorname{Rad}\left(w_{\mathrm{N}}^{2}\right)=\operatorname{St}_{N}\left(w_{\mathrm{N}}^{2}\right)=\operatorname{St}_{N}\left(w_{N^{2}}\right),
$$

whence, using Step 2, we obtain that $\mathrm{D}=\mathrm{St}_{B}(\mu)$. Put $\mathrm{E}=\mathrm{D}+\sum_{i=1}^{\infty} \frac{1}{4^{i}}\left[\mathrm{D},{ }_{i} \mathrm{D}\right]_{L}$. Note that $\mathrm{E}$ is a uniform $\mathbb{Z}_{2}$-Lie lattice. By Lemma $2.3 \operatorname{Rad}\left(w_{\mathrm{N}}^{2}\right)$ is uniform. Hence, by Lemma 2.1(2),

$$
\left[\mathrm{D}, i_{i} \mathrm{D}\right]_{L} \leq\left[\operatorname{Rad}\left(w_{\mathrm{N}}^{2}\right),_{i} \operatorname{Rad}\left(w_{\mathrm{N}}^{2}\right)\right]_{L}+2\left[\mathrm{~B},{ }_{i} \mathrm{~B}\right]_{L} \leq 4^{i}\left(\operatorname{Rad}\left(w_{\mathrm{N}}^{2}\right)+\mathrm{B}\right) .
$$

Therefore, $E \leq B$. Thus, $E^{2} \leq A \leq C \leq \operatorname{St}_{B}(\mu) \leq E$, and so $C$ is $1 \frac{1}{2}$-uniform.

Let $a$ be such that $w\left([a, \mathrm{C}]_{L}\right)=1$. Since B polarizes $w^{4}, a \in \mathrm{B}$. Hence $a \in$ $\operatorname{St}_{B}\left(w_{A}\right)=\operatorname{St}_{B}(\mu)$. Now, for every $x \in C$

$$
\delta\left([a, x]_{G}\right)=\mu\left([a, x]_{G}\right)=\mu\left([a, x]_{L}\right)=w\left([a, x]_{L}\right)=1 .
$$

Thus, $a$ fixes $\delta$ and so $a \in C=\operatorname{St}_{B}(\delta)$.

Step 7. Let $\Delta$ be the $N$-orbit of $w$ in $\operatorname{Irr}(\mathrm{N})$. Then $\phi(x)=\Phi_{\Delta}(x)$ for every $x \in N^{2}$.

First, let us show that $\delta^{B}$ coincides with $\left(w_{C}\right)^{B}$ on $B \cap N^{2}$. Indeed, let $x \in B \cap N^{2}$. If $x \notin A$, then $x \notin C$ by Step 2. Hence $\delta^{B}(x)=\left(w_{C}\right)^{B}(x)=0$. If $x \in A$ but $x \notin \mathrm{St}_{A}\left(w_{\mathrm{B}}\right)$, then again

$$
\delta^{B}(x)=\left|\mathrm{St}_{B}(\mu): C\right| \sum_{t \in T} \mu^{t}(x)=0=\left|\mathrm{St}_{B}(\mu): C\right| \sum_{t \in T} w^{t}(x)=\left(w_{C}\right)^{B}(x) .
$$

In the case $x \in \mathrm{St}_{A}\left(w_{\mathrm{B}}\right), \delta^{B}(x)=\left(w_{C}\right)^{B}(x)$ by Lemma 2.10,

Since $\Phi_{\Delta}=\left(w_{C}\right)^{N}=\left(\left(w_{C}\right)^{B}\right)^{N}$, by Lemma 2.8 and $\phi=\delta^{N}=\left(\delta^{B}\right)^{N}$, we obtain the desired result.

Now, in order to construct the desired bijection, we have to show that the number of irreducible characters lying over $\lambda$ coincides with the number of $N$-orbits of characters $w \in \operatorname{Irr}(\mathbf{N})$ such that $\left\langle w_{N^{2}}, \lambda\right\rangle \neq 0$. In both cases this number is equal to $\frac{\left|\mathrm{St}_{B}(\mu): A\right|}{\left|\mathrm{St}_{B}(\mu): C\right|^{2}}$. 
Now we assume that $N$ is 3 -uniform. In this case we have that $\mathrm{B}$ is $2 \frac{1}{2}$-uniform. Hence $w\left([\mathrm{~B}, \mathrm{~B}, \mathrm{~B}]_{L}\right)=w\left([4 \mathrm{~B}, \mathrm{~B}]_{L}\right)=1$, and so $[B, B, B]_{G} \leq \operatorname{ker} \gamma$. Define $Z$ to be the center of $\gamma$, and let $\sigma \in \operatorname{Irr}(Z)$ be the irreducible constituent of $\gamma_{Z}$. Define a function $d$ on $Z$ by means of $d(z)=\sigma(z) w(z)^{-1}$.

Step 8. Final step.

Since $\beta$ is an extension of $\mu$ and $\mu$ coincides with $w$ on $A^{2}$, we have that $x \in Z$ if and only if $\mu\left([x, B]_{G}\right)=1$ if and only if $x \in \operatorname{St}_{B}\left(w_{B}\right)$. Thus, $Z=\operatorname{St}_{B}\left(w_{B}\right)$. Hence, by Step $2, N^{2} \cap Z=\operatorname{St}_{A}\left(w_{B}\right)$. By Lemma 2.10, $d(x)=1$ for every $x \in N^{2} \cap Z$. Now, if $a \in Z$ and $x \in N^{2} \cap Z$, then

$$
d(a x)=\sigma(a x) w(a x)^{-1}=\sigma(a) \sigma(x)\left(w(a) w(x) w\left(\left[a, \frac{1}{2} x\right]_{L}\right)\right)^{-1}=d(a) .
$$

Thus, we can extend $d$ to a function from $N / N^{2}$ to \pm 1 . Observe that

$$
\phi=\gamma^{N}=\frac{1}{|B: Z|^{1 / 2}}\left(\sigma^{B}\right)^{N}=\frac{1}{|B: Z|^{1 / 2}} \sigma^{N} \text { and } \Phi_{\Delta}=\frac{1}{|B: Z|^{1 / 2}}\left(w_{Z}\right)^{N} .
$$

Hence

$$
\Phi_{\Delta}(x)=d(x) \phi(x) \text { for every } x \in N
$$

Corollary 2.13. Let $N$ be a uniform pro-p group. Then

$$
\zeta^{N}(s)=\sum_{w \in \operatorname{Irr}(\mathrm{N})}|\mathrm{N}: \operatorname{Rad}(w)|^{\frac{1}{2}(-s-2)} .
$$

Proof. By Theorems 2.9 and 2.12, there exists a bijection $f$ between $\operatorname{Irr}(N)$ and $\left\{\Phi_{\Delta} \mid \Delta\right.$ is an $N$-orbit in $\left.\operatorname{Irr}(\mathrm{N})\right\}$ such that for any $\phi \in \operatorname{Irr}(N), f(\phi)(1)=\phi(1)$. Hence

$$
\zeta^{N}(s)=\sum_{\Delta}|\Delta|^{-\frac{1}{2} s}=\sum_{w \in \operatorname{Irr}(\mathrm{N})}\left|N: \mathrm{St}_{N}(w)\right|^{\frac{1}{2}(-s-2)}=\sum_{w \in \operatorname{Irr}(\mathrm{N})}|\mathrm{N}: \operatorname{Rad}(w)|^{\frac{1}{2}(-s-2)} .
$$

\section{3. $p$-ADIC INTEGRATION}

In this section we give an explanation of the notion of a definable $p$-adic integral and we introduce the facts that we will use later. More detailed discussion of this subject can be found in [3, 6, 4, 8,

We use the standard notation for $p$-adic sets. ||$_{p}$ is the standard $p$-adic valuation on $\mathbb{Q}_{p}$ : if $a \in p^{k} \mathbb{Z}_{p} \backslash p^{k+1} \mathbb{Z}_{p}$, then $|a|_{p}=p^{-k}$. Also, $\mu$ will be the Haar measure on $\mathbb{Q}_{p}^{n}$. We always suppose that $\mu\left(\mathbb{Z}_{p}^{n}\right)=1$.

Let $\mathbf{X}=\left(X_{1}, \cdots, X_{M}\right)$ be $M$ commuting indeterminates, and let $\mathbb{Q}_{p}[[\mathbf{X}]]$ denote the set of formal power series over $\mathbb{Q}_{p}$. We define the following subsets of $\mathbb{Q}_{p}[[\mathbf{X}]]$ :

(1) $\mathbb{Z}_{p}[[\mathbf{X}]]$ denotes the set of power series over $\mathbb{Z}_{p}$;

(2) $\mathbb{Q}_{p}\{\mathbf{X}\}$ consists of all formal power series $\sum_{i \in \mathbb{N}_{\geq 0}^{M}} a_{i} \mathbf{X}^{i}$ such that $\left|a_{i}\right|_{p} \rightarrow 0$ as $|i| \rightarrow \infty$.

(3) $\mathbb{Z}_{p}\{\mathbf{X}\}=\mathbb{Z}_{p}[[\mathbf{X}]] \cap \mathbb{Q}_{p}\{\mathbf{X}\}$.

We define the function $D: \mathbb{Z}_{p}^{2} \rightarrow \mathbb{Z}_{p}$ by

$$
D(x, y)= \begin{cases}x / y & \text { if }|x|_{p} \leq|y|_{p} \text { and } y \neq 0, \\ 0 & \text { otherwise. }\end{cases}
$$


For $n>0$ we define $P_{n}$ to be the set of nonzero $n$th powers in $\mathbb{Z}_{p}$.

We define the following language considered in [6]:

Let $\mathrm{L}_{\mathrm{D}}^{\text {an }}$ be the language with logical symbols $\forall, \exists, \neg, \vee, \&,=$, a countable number of variables $X_{i}$ and

(1) an $m$-place operation symbol $F$ for each $F(\mathbf{X}) \in \mathbb{Z}_{p}\{\mathbf{X}\}, m \geq 0$;

(2) a binary operation symbol $D$;

(3) a unitary relation symbol $P_{n}$ for each $n \geq 2$.

Each formula $\phi\left(x_{1}, \cdots, x_{M}\right)$ in the language $\mathrm{L}_{\mathrm{D}}^{\text {an }}$ defines a subset

$$
M_{\phi}=\left\{\mathbf{x} \in \mathbb{Z}_{p}^{M} \mid \phi(\mathbf{x}) \text { is true in } \mathbb{Z}_{p}\right\}
$$

where we interpret

(1) each $F \in \mathbb{Z}_{p}\{\mathbf{X}\}$ as a function $f: \mathbb{Z}_{p}^{n} \rightarrow \mathbb{Z}_{p}$ defined by $f(\mathbf{x})=F(\mathbf{x})$;

(2) the binary operation symbol $D$ as the function $D$;

(3) $P_{n}(x)$ to be true if $x \in P_{n}$.

We call such a subset $M_{\phi}$ definable. A function $f: V \rightarrow \mathbb{Z}_{p}$ is called definable if its graph is a definable subset. Note that, in particular, a definable function is bounded.

With each pair of definable functions $f_{1}: \mathbb{Z}_{p}^{M} \rightarrow \mathbb{Z}_{p}, f_{2}: \mathbb{Z}_{p}^{M} \rightarrow \mathbb{Z}_{p}$ and each definable subset $U$ of $\mathbb{Z}_{p}^{M}$ we associate the following function:

$$
I\left(f_{1}, f_{2}, U, s\right)=\int_{U}\left|f_{1}(\mathbf{x})\right|_{p}^{s}\left|f_{2}(\mathbf{x})\right|_{p} d \mu .
$$

We shall call this function a definable integral. Our proof of Theorem 1.1 is based on the following important result:

Theorem 3.1. Suppose that $I\left(f_{1}, f_{2}, U, s\right)$ is a definable integral. Then

(1) $U$ is measurable, and

(2) $I\left(f_{1}, f_{2}, U, s\right)$ is a rational function in $p^{-s}$.

In this form Theorem 3.1 appears in [8, Theorem 1.8] (see also [5, Theorem 2.6]). Its proof is presented in [6] and [4.

\section{THE UNIFORM CASE}

In this section we prove Theorem 1.2 First, we consider the case $g=1$, and then we present a general argument. The main steps of our proof are as follows. First, using Corollary 2.13 we interpret the function $\zeta^{N}(s)$ in terms of the associated $\mathbb{Z}_{p}$-Lie lattice. Next, we express the function

$$
\zeta(s)=\sum_{w \in \operatorname{Irr}(\mathrm{N})}|\mathrm{N}: \operatorname{Rad}(w)|^{-s}
$$

as a definable $p$-adic integral and use Theorem 3.1

Recall that $\mathrm{N}^{*}=\operatorname{Hom}_{\mathbb{Z}_{p}}\left(\mathrm{~N}, \mathbb{Z}_{p}\right)$. Since $N$ acts on $\mathrm{N}, \mathrm{N}^{*}$ is also an $N$-module. For each $i \geq 0$ we fix $\theta_{i}$ a $p^{i}$ th primitive root of 1 in $\mathbb{C}$ and we construct an $N$-homomorphism $\pi_{p^{i}}: \mathrm{N}^{*} \rightarrow \operatorname{Irr}\left(\mathrm{N} / p^{i} \mathrm{~N}\right)$ in the following way:

$$
\pi_{p^{i}}(m)\left(l+p^{i} \mathrm{~N}\right)=\theta_{i}^{m(l)}
$$

The kernel of $\pi_{p^{i}}$ is equal to $p^{i} \mathrm{~N}^{*}$, and so $\pi_{p^{i}}$ is surjective. For any $0 \neq z \in \mathbb{Z}_{p}$ such that $|z|_{p}=p^{-i}$ we define $\pi_{z}=\pi_{p^{i}}$. Put $W=\left(\mathbf{N}^{*} \backslash p \mathbf{N}^{*}\right) \times\left(p \mathbb{Z}_{p} \backslash\{0\}\right)$. This 
set will be the domain of integration:

Lemma 4.1. Let $\beta(a, z)=\left|\mathrm{N}: \operatorname{Rad}\left(\pi_{z}(a)\right)\right|$, where $(a, z) \in W$. Denote by $n$ the $\mathbb{Z}_{p}$-rank of $\mathrm{N}$. Then

$$
\zeta(s)=1+p(p-1)^{-1} \int_{W}|z|_{p}^{-(n+1)}|\beta(a, z)|_{p}^{s} d a d z .
$$

Proof. First note that if $(a, z) \in W$ and $w=\pi_{z}(a)$, then $|z|_{p}=o(w)^{-1}$. Hence

$$
\mu\left(\left\{(a, z) \in W \mid \pi_{z}(a)=w\right\}\right)=(p-1) p^{-1} o(w)^{-(n+1)} .
$$

Therefore

$$
\begin{aligned}
\zeta(s)-1 & =\sum_{1_{\mathrm{N}} \neq w \in \operatorname{Irr}(\mathrm{N})}|\mathrm{N}: \operatorname{Rad}(w)|^{-s} \\
& =\sum_{1_{\mathrm{N}} \neq w \in \operatorname{Irr}(\mathrm{N})} \int_{(a, z) \in W, \pi_{z}(a)=w} p(p-1)^{-1}|z|_{p}^{-(n+1)}\left|\mathrm{N}: \operatorname{Rad}\left(\pi_{z}(a)\right)\right|^{-s} d a d z \\
& =p(p-1)^{-1} \int_{W}|z|_{p}^{-(n+1)}\left|\mathrm{N}: \operatorname{Rad}\left(\pi_{z}(a)\right)\right|^{-s} d a d z \\
& =p(p-1)^{-1} \int_{W}|z|_{p}^{-(n+1)}|\beta(a, z)|_{p}^{s} d a d z .
\end{aligned}
$$

The integral which appears in the previous lemma is not definable. In the following we will see how we can transform it in a definable integral.

We recall now some known facts about endomorphisms of $\mathbb{Z}_{p}^{n}$. Any book on matrices over a principal ideal domain contains these facts (see, for example, [22]). Let $A, B \in \mathbb{M}_{n}\left(\mathbb{Z}_{p}\right)$ be two matrices over $\mathbb{Z}_{p}$. We write $A \sim B$ if there are two invertible matrices $C_{1}$ and $C_{2}$ over $\mathbb{Z}_{p}$ such that $C_{1} A C_{2}=B$. Of course, $A \sim B$ is an equivalence relation. The canonical forms of matrices with respect to this relation are the following:

Lemma 4.2. Let $A \in \mathbb{M}_{n}\left(\mathbb{Z}_{p}\right)$ be a matrix over $\mathbb{Z}_{p}$. Then there exist $s_{1}, \cdots, s_{n} \in$ $\mathbb{Z}_{p}$, satisfying $\left|s_{i}\right|_{p} \geq\left|s_{i+1}\right|_{p}$ for any $1 \leq i \leq n$, such that $A \sim \operatorname{diag}\left(s_{1}, \cdots, s_{n}\right)$. Moreover, if $A \sim \operatorname{diag}\left(t_{1}, \cdots, t_{n}\right)$ with $\left|t_{i}\right|_{p} \geq\left|t_{i+1}\right|_{p}$ for any $1 \leq i \leq n$, then $\left|t_{i}\right|_{p}=\left|s_{i}\right|_{p}$.

Proof. It is enough to observe that $A \sim \operatorname{diag}\left(s_{1}, \cdots, s_{n}\right)$ if and only if $\mathbb{Z}_{p}^{n} / A\left(\mathbb{Z}_{p}^{n}\right) \cong$ $\bigoplus_{i=1}^{n} \mathbb{Z}_{p} / s_{i} \mathbb{Z}_{p}$

This diagonal form is called Smith's normal form. Now we present a method of calculating Smith's normal form. If $A=\left(a_{i j}\right)_{1 \leq i, j \leq n}$ and $U, V \subseteq\{1, \cdots, n\}$, such that $|U|=|V|$, we put

$$
g_{U, V}(A)=\operatorname{det}\left[\left(a_{i j}\right)_{i \in U, j \in V}\right] .
$$

For any $1 \leq i \leq n$ we fix an order on the set of pairs $(U, V)$, where $U$ and $V$ are subsets of $\{1, \cdots, n\}$ with $i$ elements. We put $h_{0}(A)=1$, and for $1 \leq i \leq n$ we define $h_{i}(A)$ to be $g_{U, V}(A)$, where $(U, V)$ is the unique pair such that

(1) $|U|=|V|=i$

(2) for any $U^{\prime}, V^{\prime} \subseteq\{1, \cdots, n\}$ with $\left|U^{\prime}\right|=\left|V^{\prime}\right|=i$, we have either $\left|g_{U^{\prime}, V^{\prime}}(A)\right|_{p}$ $<\left|g_{U, V}(A)\right|_{p}$ or $\left|g_{U^{\prime}, V^{\prime}}(A)\right|_{p}=\left|g_{U, V}(A)\right|_{p}$ and $(U, V) \leq\left(U^{\prime}, V^{\prime}\right)$. 
Lemma 4.3 ([22, Corollary 26.1]). Let $A \in \mathbb{M}_{n}\left(\mathbb{Z}_{p}\right)$ be a matrix over $\mathbb{Z}_{p}$. Then for any $0 \leq i \leq n-1,\left|D\left(h_{i}(A), h_{i-1}(A)\right)\right|_{p} \geq\left|D\left(h_{i+1}(A), h_{i}(A)\right)\right|_{p}$ and

$$
A \sim \operatorname{diag}\left(h_{1}(A), D\left(h_{2}(A), h_{1}(A)\right), \cdots, D\left(h_{n}(A), h_{n-1}(A)\right)\right) .
$$

By the previous lemma, if $A \sim B$, then $\left|h_{i}(A)\right|_{p}=\left|h_{i}(B)\right|_{p}$. Therefore, we can speak about $\left|h_{i}(\phi)\right|_{p}$, where $\phi \in \operatorname{Hom}_{\mathbb{Z}_{p}}\left(M_{1}, M_{2}\right)$ and $M_{1} \cong M_{2} \cong \mathbb{Z}_{p}^{n}$. Thus, Lemma 4.3 implies the following result.

Lemma 4.4. Let $M_{1} \cong M_{2} \cong \mathbb{Z}_{p}^{n}$ be two $\mathbb{Z}_{p}$-modules, $0 \neq z \in \mathbb{Z}_{p}$ and $\phi \in$ $\operatorname{Hom}_{\mathbb{Z}_{p}}\left(M_{1}, M_{2}\right)$. Then $\left|M_{1} / \phi^{-1}\left(z M_{2}\right)\right|=$

$$
\left\{\begin{array}{lll}
1 & \text { if } & |z|_{p}>\left|h_{1}(\phi)\right|_{p}, \\
|z|_{p}^{-k}\left|h_{k}(\phi)\right|_{p} \quad \text { if } & \left|D\left(h_{k}(\phi), h_{k-1}(\phi)\right)\right|_{p} \geq|z|_{p}>\left|D\left(h_{k+1}(\phi), h_{k}(\phi)\right)\right|_{p}, \\
& & \quad 1 \leq k \leq n-1, \\
|z|_{p}^{-n}\left|h_{n}(\phi)\right|_{p} \quad \text { if } \quad & |z|_{p} \leq\left|D\left(h_{n}(\phi), h_{n-1}(\phi)\right)\right|_{p} .
\end{array}\right.
$$

Proof. By Lemma 4.2, we can choose bases of $M_{1}$ and $M_{2}$ such that the matrix $A$ associated with $\phi$ in these bases is diagonal and equal to

$$
\operatorname{diag}\left(h_{1}(A), D\left(h_{2}(A), h_{1}(A)\right), \cdots, D\left(h_{n}(A), h_{n-1}(A)\right)\right) .
$$

Since $\left|h_{i}(\phi)\right|_{p}=\left|h_{i}(A)\right|_{p}$ for all $i$, we obtain the lemma from Lemma 4.3 .

Let $\Psi: \mathrm{N}^{*} \rightarrow \operatorname{Hom}_{\mathbb{Z}_{p}}\left(\mathrm{~N}, \mathrm{~N}^{*}\right)$ be the map defined in the following way: if $l, k \in \mathrm{N}$ and $m \in \mathrm{N}^{*}$, then

$$
\Psi(m)(l)(k)=m\left([k, l]_{L}\right) .
$$

Lemma 4.5. Let $m \in \mathrm{N}^{*}$ and $0 \neq z \in \mathbb{Z}_{p}$. Then

$$
\operatorname{Rad}\left(\pi_{z}(m)\right)=(\Psi(m))^{-1}\left(z \mathrm{~N}^{*}\right) .
$$

Proof. Let $|z|_{p}=p^{-i}$. Then we have the following series of equivalent propositions:

$$
\begin{aligned}
& x \in \operatorname{Rad}\left(\pi_{z}(m)\right) \Leftrightarrow \pi_{z}(m)\left([y, x]_{L}\right)=1 \forall y \in \mathrm{N} \\
& \quad \Leftrightarrow \theta_{i}^{m\left([y, x]_{L}\right)}=1 \forall y \in \mathrm{N} \Leftrightarrow m\left([x, y]_{L}\right) \in z \mathbb{Z}_{p} \forall y \in \mathrm{N} \\
& \quad \Leftrightarrow \Psi(m)(x)(y) \in z \mathbb{Z}_{p} \forall y \in \mathrm{N} \Leftrightarrow \Psi(m)(x) \in z \mathrm{~N}^{*} \Leftrightarrow x \in(\Psi(m))^{-1}\left(z \mathrm{~N}^{*}\right) .
\end{aligned}
$$

Let $\left\{e_{1}, \cdots, e_{n}\right\}$ be a basis of $\mathrm{N}$ and $\left\{f_{1}, \cdots, f_{n}\right\}$ a basis of $\mathrm{N}^{*}$. Thus, any element $a$ from $\mathrm{N}$ or $\mathrm{N}^{*}$ is identified with a vector $\left(a_{1}, \cdots, a_{n}\right)$, and we can view $\operatorname{Hom}_{\mathbb{Z}_{p}}\left(\mathrm{~N}, \mathrm{~N}^{*}\right)$ as $\mathbb{M}_{n}\left(\mathbb{Z}_{p}\right)$. Then the entries of $\Psi(a), a \in \mathrm{N}^{*}$, are linear functions on $\left\{a_{i}\right\}$ and $g_{U, V}(\Psi(a))$ are polynomials on $\left\{a_{i}\right\}$ for every $U, V \subseteq\{1, \cdots, n\}$ with $|U|=|V|$. This implies that the functions $h_{i}(\Psi(a))$ are definable in $\mathrm{L}_{\mathrm{D}}^{\mathrm{an}}$.

Define the following subsets of $W$ :

$$
W_{0}=\left\{\left.(a, z) \in W|| z\right|_{p}>\left|h_{1}(\Psi(a))\right|_{p}\right\} ;
$$

for any $1 \leq k \leq n-1$,

$$
\begin{aligned}
W_{k}=\left\{( a , z ) \in W | | D \left(h_{k}(\Psi(a)),\right.\right. & \left.h_{k-1}(\Psi(a))\right)\left.\right|_{p} \geq|z|_{p} \\
& \left.>\left|D\left(h_{k+1}(\Psi(a)), h_{k}(\Psi(a))\right)\right|_{p}\right\} ;
\end{aligned}
$$

and

$$
W_{n}=\left\{\left.(a, z) \in W|| z\right|_{p} \leq\left|D\left(h_{n}(\Psi(a)), h_{n-1}(\Psi(a))\right)\right|_{p}\right\} .
$$

If $(a, z) \in W_{k}$ define $\alpha(a, z)=D\left(z^{k}, h_{k}(\Psi(a))\right)$. Then $\alpha$ is a definable function on $W$. Using Lemmas 4.4 and 4.5 , we obtain the following corollary. 
Corollary 4.6. Let $(a, z) \in W$. Then $\left|\mathrm{N}: \operatorname{Rad}\left(\pi_{z}(a)\right)\right|=|\alpha(a, z)|_{p}^{-1}$.

Now, suppose that $N$ is FAb. In this case we have the following important property.

Lemma 4.7. Let $N$ be an FAb uniform pro-p group. Then the number

$$
m(\mathrm{~N})=\min \left\{k \mid p^{k} \mathrm{~N} \subseteq[\mathrm{N}, \mathrm{N}]_{L}\right\}
$$

is finite and for any $w \in \operatorname{Irr}(\mathrm{N})$ we have $o(w) \leq|\mathrm{N}: \operatorname{Rad}(w)| p^{m(\mathrm{~N})}$.

Proof. Since $N$ is FAb, $[\mathrm{N}, \mathrm{N}]_{L}$ is of finite index in $\mathrm{N}$. Thus, $m(\mathrm{~N})$ is finite.

Since $p^{m(\mathrm{~N})} \mathrm{N} \leq[\mathrm{N}, \mathrm{N}]_{L}$, we obtain that

$$
|\mathrm{N}: \operatorname{Rad}(w)| p^{m(\mathrm{~N})} \mathbf{N} \leq[\mathbf{N},|\mathbf{N}: \operatorname{Rad}(w)| \mathbf{N}]_{L} \leq[\mathrm{N}, \operatorname{Rad}(w)]_{L} .
$$

Hence

$$
w\left(|\mathbf{N}: \operatorname{Rad}(w)| p^{m(\mathrm{~N})} \mathbf{N}\right)=1,
$$

and so $|\mathbf{N}: \operatorname{Rad}(w)| p^{m(\mathrm{~N})} \geq o(w)$.

Corollary 4.8. Let $(a, z) \in W$. Then

$$
\left|p^{m(\mathrm{~N})} \alpha(a, z)\right|_{p} \leq|z|_{p}
$$

Proof. Note that if $w=\pi_{z}(a)$, then $|z|_{p}=o(w)^{-1}$. Therefore, by Lemma 4.7 and Corollary 4.6, $|z|_{p}^{-1} \leq|\alpha(a, z)|_{p}^{-1} p^{m(\mathrm{~N})}$. Hence $\left|p^{m(\mathrm{~N})} \alpha(a, z)\right|_{p} \leq|z|_{p}$.

Theorem 4.9. Let $N$ be an FAb uniform pro-p group. Then $\zeta^{N}(s)$ is a rational function in $p^{-s}$.

Proof. Let $n$ be the $\mathbb{Z}_{p}$-rank of N. By Lemma 4.1 and Corollary 4.6

$$
\zeta(s)-1=p(p-1)^{-1} \int_{W}|z|_{p}^{-(n+1)}|\alpha(a, z)|_{p}^{s} d a d z .
$$

By Corollary 4.8, we have that if $(a, z) \in W$, then

$$
\begin{aligned}
|z|_{p}^{-1} & =|z|_{p}^{-1}\left|p^{m(\mathrm{~N})} \alpha(a, z)\right|_{p}\left|p^{m(\mathrm{~N})} \alpha(a, z)\right|_{p}^{-1} \\
& =\left|D\left(p^{m(\mathrm{~N})} \alpha(a, z), z\right)\right|_{p}\left|p^{m(\mathrm{~N})} \alpha(a, z)\right|_{p}^{-1} .
\end{aligned}
$$

Therefore, we have

$$
\begin{aligned}
& \int_{W}|z|_{p}^{-(n+1)}|\alpha(a, z)|_{p}^{s} d a d z \\
& \quad=p^{m(\mathrm{~N})(n+1)}\left(\int_{W}\left|D\left(p^{m(\mathrm{~N})} \alpha(a, z), z\right)\right|_{p}^{n+1}|\alpha(a, z)|_{p}^{s-n-1} d a d z\right) .
\end{aligned}
$$

Now, from Theorem 3.1 we obtain that the last integral is a rational function in $p^{-s}$, whence $\zeta(s)$ is a rational function in $p^{-s}$. Note that $\zeta(s)$ is a function in $p^{-2 s}$, whence $\zeta(s)$ is a rational function in $p^{-2 s}$. Thus, by Corollary 2.13, $\zeta^{N}(s)$ is a rational function in $p^{-s}$.

We dedicate the rest of this section to the proof of Theorem 1.2. The idea of the proof is the same as in the proof of the previous theorem.

Lemma 4.10. Let $N$ be an FAb uniform pro-p group and $\mu_{i}$ the sum of all irreducible characters of $N$ of degree $p^{i}$. Then $\mu_{i}$ is equal to the sum of all $\Phi_{\Delta}$ where $\Delta$ is any $N$-orbit in $\operatorname{Irr}(\mathrm{N})$ of size $p^{2 i}$. 
Proof. Denote by $\rho_{i}$ the sum of all $\Phi_{\Delta}$ where $\Delta$ is any $N$-orbit in $\operatorname{Irr}(\mathrm{N})$ of size $p^{2 i}$. Then if $p>2$, Theorem 2.9 says that $\mu_{i}=\rho_{i}$.

If $p=2$, Theorem 2.12 says that $\mu_{i}$ coincides with $\rho_{i}$ on elements from $N^{2}$. Now, note that for any character $\sigma$ of $N / N^{2}, \rho_{i}=\sigma \rho_{i}$ and $\mu_{i}=\sigma \mu_{i}$. Hence both $\rho_{i}$ and $\mu_{i}$ vanish outside $N^{2}$. We conclude that $\rho_{i}=\mu_{i}$ in the case $p=2$ as well.

Now we are ready to prove Theorem 1.2 .

Theorem 4.11. Let $N$ be a uniform pro-p group. Then for any $g \in N$,

$$
\sum_{i=0}^{\infty} \mu_{i}(g) p^{-s i}=\sum_{\lambda \in \operatorname{Irr}(G)} \lambda(g) \lambda(1)^{-s}
$$

is a rational function in $p^{s}$.

Proof. By the previous lemma,

$$
\sum_{i=0}^{\infty} \mu_{i}(g) p^{-s i}=\sum_{w \in \operatorname{Irr}(\mathrm{N})} w(g)|\mathrm{N}: \operatorname{Rad}(w)|^{-(s+1) / 2} .
$$

Let $\theta_{m}$ be a $p^{m}$ th primitive root of 1 . If the order of $w$ is equal to $p^{m} \geq p$, then

$$
\sum_{\sigma \in \operatorname{Gal}\left(\mathbb{Q}\left(\theta_{m}\right) / \mathbb{Q}\right)} w^{\sigma}(g)=\left\{\begin{array}{lll}
(p-1) p^{m-1} & \text { if } & g \in \operatorname{Ker} w \\
-p^{m-1} & \text { if } & o(w(g))=p, \\
0 & \text { if } & o(w(g))>p .
\end{array}\right.
$$

Therefore, we have

$$
\begin{aligned}
\sum_{i=0}^{\infty} \mu_{i}(g) p^{-s i}= & \sum_{w \in \operatorname{Irr}(\mathrm{N}), o(w(g))=1}|\mathrm{~N}: \operatorname{Rad}(w)|^{-(s+1) / 2} \\
& -\frac{1}{p-1} \sum_{w \in \operatorname{Irr}(\mathrm{N}), o(w(g))=p}|\mathrm{~N}: \operatorname{Rad}(w)|^{-(s+1) / 2} .
\end{aligned}
$$

Now, note that the sets $V_{1}=\{(a, z) \in W \mid a(g) \equiv 0(\bmod z)\}$ and $V_{2}=\{(a, z) \in$ $W \mid p a(g) \equiv 0(\bmod z)\}$ are definable. Following the proof of Theorem 4.9, we obtain that

$$
\sum_{w \in \operatorname{Irr}(\mathbf{N}), o(w(g))=1}|\mathbf{N}: \operatorname{Rad}(w)|^{-s}-1=p(p-1)^{-1} \int_{V_{1}}|z|_{p}^{-(n+1)}|\alpha(a, z)|_{p}^{s} d a d z,
$$

and

$$
\sum_{w \in \operatorname{Irr}(\mathbf{N}), o(w(g))=p}|\mathrm{~N}: \operatorname{Rad}(w)|^{-s}=p(p-1)^{-1} \int_{V_{2} \backslash V_{1}}|z|_{p}^{-(n+1)}|\alpha(a, z)|_{p}^{s} d a d z .
$$

Hence, as in the proof of Theorem 4.9 we conclude that $\sum_{i=0}^{\infty} \mu_{i}(g) p^{-s i}$ is a rational function in $p^{-s}$.

\section{Character extensions}

In order to prove Theorem 1.1 for an arbitrary compact $p$-adic analytic group $G$, we need to develop a theory of extensions of characters from a normal uniform subgroup $N$ of $G$ to characters of $G$. Thus, we should adapt the Clifford theory to our case. Let $F$ be a profinite group, $V$ a normal open subgroup of $F$, and $\theta \in \operatorname{Irr}(V)$ an irreducible character of $V$. Under these hypotheses we say that 
$(F, V, \theta)$ is a generalized character triple. If, moreover, $\theta$ is $F$-invariant, then we say that $(F, V, \theta)$ is a character triple. We refer the reader to [14, Section 11] for general discussion on character triples. For every generalized character triple we define the function $f_{(F, V, \theta)}(s)$ by means of

$$
f_{(F, V, \theta)}(s)=\sum_{\lambda \in \operatorname{Irr}(F \mid \theta)}\left(\frac{\lambda(1)}{\theta(1)}\right)^{-s} .
$$

Thus, for example, if $N$ is an open normal subgroup of $G$, we have the following expression for $\zeta^{G}(s)$ :

$$
\zeta^{G}(s)=\sum_{\theta \in \operatorname{Irr}(N)} \frac{\theta(1)^{-s} f_{(G, N, \theta)}(s)}{\left|G: \operatorname{St}_{G}(\theta)\right|} .
$$

From the Clifford theory we know that if $(F, V, \theta)$ is a generalized character triple, then

$$
f_{(F, V, \theta)}(s)=\left|F: \operatorname{St}_{F}(\theta)\right|^{-s} f_{\left(\mathrm{St}_{F}(\theta), V, \theta\right)}(s) .
$$

Therefore, the calculation of the function $f_{(F, V, \theta)}(s)$ is reduced to the case when $(F, V, \theta)$ is a character triple. From now on we will suppose this.

For the convenience of the reader and in order to fix the notation, we recall the definition of the abelian group $H^{2}\left(K, \mathbb{C}^{*}\right)$, where $K$ is an arbitrary group. A 2-cocycle of $K$ is a function $\alpha: K \times K \rightarrow \mathbb{C}^{*}$ such that

$$
\alpha(x y, z) \alpha(x, y)=\alpha(x, y z) \alpha(y, z) .
$$

The set of 2-cocycles of $K$ forms a group under pointwise multiplication. This group is denoted by $Z^{2}\left(K, \mathbb{C}^{*}\right)$. If $\mu: K \rightarrow \mathbb{C}^{*}$ is an arbitrary function, we can define $\delta(\mu): K \times K \rightarrow \mathbb{C}^{*}$ by

$$
\delta(\mu)(g, h)=\mu(g) \mu(h) \mu(g h)^{-1} .
$$

Note that $\delta$ is a homomorphism from the group of $\mathbb{C}^{*}$-valued functions on $K$ into $Z^{2}\left(K, \mathbb{C}^{*}\right)$. The image of $\delta$ is the subgroup $B^{2}\left(K, \mathbb{C}^{*}\right) \leq Z^{2}\left(K, \mathbb{C}^{*}\right)$ which is called the group of 2-coboundaries. The factor group $Z^{2}\left(K, \mathbb{C}^{*}\right) / B^{2}\left(K, \mathbb{C}^{*}\right)$ is denoted by $H^{2}\left(K, \mathbb{C}^{*}\right)$.

If $K_{1}$ and $K_{2}$ are two groups and $\phi: K_{1} \rightarrow K_{2}$ is a group homomorphism, then $\phi$ induces naturally a homomorphism $\phi^{*}: Z^{2}\left(K_{2}, \mathbb{C}^{*}\right) \rightarrow Z^{2}\left(K_{1}, \mathbb{C}^{*}\right)$ by means of

$$
\phi^{*}(\alpha)(g, h)=\alpha(\phi(g), \phi(h)), \alpha \in Z^{2}\left(K_{2}, \mathbb{C}^{*}\right), g, h \in K_{1} .
$$

Since $\phi^{*}\left(B^{2}\left(K_{2}, \mathbb{C}^{*}\right)\right) \leq B^{2}\left(K_{1}, \mathbb{C}^{*}\right)$, we obtain a homomorphism $H^{2}\left(K_{2}, \mathbb{C}^{*}\right) \rightarrow$ $H^{2}\left(K_{1}, \mathbb{C}^{*}\right)$, which we will also denote by $\phi^{*}$.

From [14, Theorem 11.7] we know that any character triple $(F, V, \theta)$ determines uniquely an element from $H^{2}\left(F / V, \mathbb{C}^{*}\right)$. We denote this element by $\beta_{(F, V, \theta)}$. Let us recall this construction. Let $T$ be a transversal for $V$ in $F$ and $M$ a left irreducible $\mathbb{C} V$-module corresponding to the character $\theta$. Since $\theta$ is $F$-stable, the left $\mathbb{C} V$ module $t M$ is isomorphic to $M$ for any $t \in T$. Let $\psi_{t}: t M \rightarrow M$ be an isomorphism of $\mathbb{C} V$-modules. Put $a_{t}(m)=\psi_{t}(t m)$. Then it is clear that $a_{t} \in \operatorname{End}_{\mathbb{C}}(M)$. Any element $f \in F$ has the form $f=t n$, where $t \in T$ and $n \in N$. We define an endomorphism $a_{f}$ of $M$ by means of $a_{f}(m)=a_{t}(n m)$. Let $g \in V, m \in M$, and $f_{1}, f_{2} \in F$. From the construction of $a_{f}$ we have

$$
a_{\left(f_{1} f_{2}\right)^{-1}} a_{f_{1}} a_{f_{2}}(g m)=g a_{\left(f_{1} f_{2}\right)^{-1}} a_{f_{1}} a_{f_{2}}(m) .
$$


Hence $a_{\left(f_{1} f_{2}\right)^{-1}} a_{f_{1}} a_{f_{2}}$ is an endomorphism of $M$ as a $\mathbb{C} V$-module. But $M$ is irreducible and so, by Schur's Lemma, $a_{\left(f_{1} f_{2}\right)^{-1}} a_{f_{1}} a_{f_{2}}$ is multiplication by a nonzero constant, which we denote by $\alpha_{\left(F, V, \theta, T,\left\{\psi_{t}\right\}\right)}\left(f_{1} V, f_{2} V\right)$. Now, it can be proved that $\alpha_{\left(F, V, \theta, T,\left\{\phi_{t}\right\}\right)}$ is a 2-cocycle of $F / V$; its image in $H^{2}\left(F / V, \mathbb{C}^{*}\right)$ is denoted by $\beta_{(F, V, \theta)}$. Although the construction of $\alpha_{\left(F, V, \theta, T,\left\{\psi_{f}\right\}\right)}$ depends on the choice of $T$ and $\psi_{t}, t \in T$, the element $\beta_{(F, V, \theta)}$ does not.

For our purposes the following proposition is very useful.

Proposition 5.1. Let $\left(F_{i}, V_{i}, \theta_{i}\right)(i=1,2)$ be two character triples where $V_{i}$ $(i=1,2)$ are pro-p groups, and let $P_{i}$ be a pro-p Sylow subgroup of $F_{i}$. If $\phi$ : $F_{1} / V_{1} \rightarrow F_{2} / V_{2}$ is an isomorphism such that $\phi\left(P_{1} / V_{1}\right)=P_{2} / V_{2}$ and $\beta_{\left(P_{1}, V_{1}, \theta_{1}\right)}$ $=\phi^{*}\left(\beta_{\left(P_{2}, V_{2}, \theta_{2}\right)}\right)$, then $f_{\left(F_{1}, V_{1}, \theta_{1}\right)}=f_{\left(F_{2}, V_{2}, \theta_{2}\right)}$.

Proof. First, note that by [23, Corollary 7.26(3)], $\beta_{\left(F_{1}, V_{1}, \theta_{1}\right)}=\phi^{*}\left(\beta_{\left(F_{2}, V_{2}, \theta_{2}\right)}\right)$. Then the argument of [14. Theorem 11.28] implies that $\left(F_{1}, V_{1}, \theta_{1}\right)$ and $\left(F_{2}, V_{2}, \theta_{2}\right)$ are isomorphic character triples in the sense of [14, Definition 11.23]. Hence, by [14, Lemma 11.24], $f_{\left(F_{1}, V_{1}, \theta_{1}\right)}=f_{\left(F_{2}, V_{2}, \theta_{2}\right)}$.

The following result is a generalization of [15, Lemma 4.1].

Lemma 5.2. Let $(F, V, \theta)$ be a character triple and $S$ a subgroup of $F$ such that $F=S V$. Put $H=V \cap S$. Assume that $\mu$ is an irreducible $S$-stable character of $H$ and $\left\langle\mu, \theta_{H}\right\rangle=1$. Then $\tau^{*}\left(\beta_{(F, V, \theta)}\right)=\beta_{(S, H, \mu)}$, where $\tau: S / H \rightarrow F / V$ is the natural group isomorphism that sends sH to $\mathrm{sV}$.

Proof. Let $M$ be a left irreducible $\mathbb{C} V$-module corresponding to the character $\theta$. Then the restriction of $M$ to $H$ is isomorphic to $\bigoplus M_{i}$. Since $\left\langle\mu, \theta_{H}\right\rangle=1$, we can suppose that $M_{1}$ corresponds to the character $\mu$ and the rest of the $M_{i}$ are not isomorphic to $M_{1}$. Let $T$ be a transversal for $H$ in $S$. Then $T$ is also a transversal for $V$ in $F$. As was explained at the beginning of this section, for any $t \in T$ we can define an isomorphism of $V$-modules $\psi_{t}: t M \rightarrow M$. We show that $\psi_{t}$ sends $t M_{1}$ to $M_{1}$. Since $t M_{1}$ is isomorphic to $M_{1}$ as an $H$-module, because $t$ fixes $\mu$ and $\psi_{t}$ is a $V$-isomorphism, $\psi_{t}\left(t M_{1}\right)$ is isomorphic to $M_{1}$ as an $H$-module. Since $M$ has only one $H$-submodule isomorphic to $M_{1}$, we obtain that $\psi_{t}\left(t M_{1}\right)=M_{1}$. Hence $\left(\psi_{t}\right)_{t M_{1}}: t M_{1} \rightarrow M_{1}$ is an isomorphism which we can use in the calculation of $\beta(S, H, \mu)$. Thus, for any $x, y \in S$ and $0 \neq m \in M_{1}$ we have

$$
\begin{aligned}
\tau^{*}\left(\alpha_{\left(F, V, \theta, T,\left\{\psi_{t}\right\}\right)}\right)(x H, y H) & =\alpha_{\left(F, V, \theta, T,\left\{\psi_{t}\right\}\right)}(x V, y V) \\
& =\frac{\psi_{x y}^{-1}\left((x y)^{-1} \psi_{x}\left(x \psi_{y}(y m)\right)\right)}{m} \\
& =\alpha_{\left(S, H, \mu, T,\left\{\left(\psi_{t}\right)_{t M_{1}}\right\}\right)}(x H, y H) .
\end{aligned}
$$

Thus, $\tau^{*}\left(\beta_{(F, V, \theta)}\right)=\beta_{(S, H, \mu)}$.

In the following corollaries we present two particular cases of the previous lemma of special interest.

Corollary 5.3. Let $(F, V, \theta)$ be a character triple and $S$ a subgroup of $F$ such that $F=S V$. Put $H=V \cap S$. Assume that $\mu$ is an irreducible $S$-stable character of $H$ and $\mu^{V}=\theta$. Then $\tau^{*}\left(\beta_{(F, V, \theta)}\right)=\beta_{(S, H, \mu)}$, where $\tau: S / H \rightarrow F / V$ is the natural group isomorphism that sends $s H$ to $s V$.

Proof. Since $\theta$ is irreducible, $\left\langle\mu, \theta_{H}\right\rangle=\left\langle\mu^{H}, \theta\right\rangle=1$ by Frobenius reprocity. Hence we can apply Lemma 5.2 
Corollary 5.4. Let $(F, V, \theta)$ be a character triple and $S$ a subgroup of $F$ such that $F=S V$. Put $H=V \cap S$. Assume that $\mu$ is an irreducible $S$-stable character of $H$ and $\mu=\theta_{H}$. Then $\tau^{*}\left(\beta_{(F, V, \theta)}\right)=\beta_{(S, H, \mu)}$, where $\tau: S / H \rightarrow F / V$ is the natural group isomorphism that sends $s H$ to $\mathrm{sV}$.

Proof. Since $\mu$ is irreducible, $\left\langle\mu, \theta_{H}\right\rangle=1$. Hence we can apply Lemma 5.2 .

Theorem 5.5. Let $G$ be a compact $p$-adic group, $p>2, N$ a normal open 2-uniform subgroup of $G$, and $\lambda \in \operatorname{Irr}(N)$. If $w \in \operatorname{Irr}(\mathrm{N})$ and $\langle\lambda, w\rangle \neq 0$, then

(1) $\operatorname{St}_{G}(\lambda)=\operatorname{St}_{G}(w) N$,

(2) $f_{\left(\operatorname{St}_{G}(\lambda), N, \lambda\right)}(s)=f_{\left(\operatorname{St}_{G}(w), \operatorname{St}_{N}(w), w_{\mathrm{St}_{N}(w)}\right)}(s)$.

Proof. Let $\Omega$ be an $N$-orbit of $w$ in $\operatorname{Irr}(\mathrm{N})$. By Theorem 2.9, $\lambda=\Phi_{\Omega}$. Hence, it is clear that $\operatorname{St}_{G}(w) N$ stabilizes $\lambda$. On the other hand, if $\lambda^{g}=\lambda$ for some $g \in G$, then there should exist $n \in N$ such that $w^{g}=w^{n}$. Hence $g n^{-1} \in \operatorname{St}_{G}(w)$. This implies that $\operatorname{St}_{G}(\lambda) \leq \operatorname{St}_{G}(w) N$.

Let $P$ be a pro- $p$ Sylow subgroup of $\operatorname{St}_{G}(\lambda)$. From the previous paragraph $P=\operatorname{St}_{P}(w) N$. By Lemma 2.5, there exists a polarizing uniform $\mathbb{Z}_{p}$-Lie lattice A for $w$ that is stable under $\operatorname{St}_{P}(w)$. By Lemma 2.6 $w_{A}$ is a linear character of $A$.

Since $\operatorname{St}_{P}(w) \cap N=\operatorname{St}_{N}(w)=\operatorname{Rad}(w) \leq A$, we obtain that $\operatorname{St}_{P}(w) A \cap N=A$. By Lemma 2.8, $\lambda=\Phi_{\Omega}=w_{A}^{N}$. Using Corollary 5.3, we obtain that

$$
\tau_{1}^{*}\left(\beta_{(P, N, \lambda)}\right)=\beta_{\left(\operatorname{St}_{P}(w) A, A, w_{A}\right)},
$$

where $\tau_{1}: \operatorname{St}_{P}(w) A / A \rightarrow P / N$ is defined by $\tau_{1}(x A)=x N, x \in \operatorname{St}_{P}(w) A$.

Note that $\operatorname{St}_{P}(w) \cap A=\operatorname{St}_{N}(w)$. Hence by Corollary 5.4 .

$$
\tau_{2}^{*}\left(\beta_{\left(\operatorname{St}_{P}(w) A, A, w_{A}\right)}\right)=\beta_{\left(\operatorname{St}_{P}(w), \operatorname{St}_{N}(w), w_{\mathrm{St}_{N}(w)}\right)},
$$

where $\tau_{2}: \operatorname{St}_{P}(w) / \operatorname{St}_{N}(w) \rightarrow \operatorname{St}_{P}(w) A / A$ is defined by $\tau_{2}\left(x \operatorname{St}_{N}(w)\right)=x A, x \in$ $\operatorname{St}_{P}(w)$.

Let $\phi: \operatorname{St}_{G}(w) / \operatorname{St}_{N}(w) \rightarrow \operatorname{St}_{G}(\lambda) / N$ be defined by $\phi\left(x \operatorname{St}_{N}(w)\right)=x N$. Thus, $\phi_{\mathrm{St}_{P}(w) / \mathrm{St}_{N}(w)}=\tau_{1} \circ \tau_{2}$. Hence we obtain that

$$
\phi^{*}\left(\beta_{(P, N, \lambda)}\right)=\left(\tau_{1} \circ \tau_{2}\right)^{*}\left(\beta_{(P, N, \lambda)}\right)=\beta_{\left(\operatorname{St}_{P}(w), \operatorname{St}_{N}(w), w_{\mathrm{St}_{N}(w)}\right)},
$$

and so, by Proposition 5.1, $f_{\left(\mathrm{St}_{G}(\lambda), N, \lambda\right)}(s)=f_{\left(\operatorname{St}_{G}(w), \mathrm{St}_{N}(w), w_{\mathrm{St}_{N}(w)}\right)}(s)$.

Thus, the last theorem reduces the study of $f_{\left(\operatorname{St}_{G}(\lambda), N, \lambda\right)}(s)$ to the study of $f_{\left(\mathrm{St}_{G}(w), \mathrm{St}_{N}(w), w_{\mathrm{St}_{N}(w)}\right)}(s)$. The advantage of this reduction is that $w_{\mathrm{St}_{N}(w)}$ is a linear character of $\operatorname{St}_{N}(w)$. We finish this section by considering character triples of linear characters.

Let $Q$ be a finite group and $F$ a free group on $|Q|$ variables (so that $F$ is generated by $\left.x_{q}, q \in Q\right)$. Define a homomorphism $\phi: F \rightarrow Q$ by $\phi\left(x_{q}\right)=q$. Let $H$ be the kernel of this homomorphism. Put $\bar{F}=F /[H, F]$ and $\bar{H}=H /[H, F]$. Since $\bar{F}$ is central by finite, the derived subgroup $\bar{F}^{\prime}$ of $\bar{F}$ is finite. Let $\beta$ and $\alpha$ be $F$ invariant linear characters of $H$. Since they are $F$-invariant, we can regard $\alpha$ and $\beta$ as characters of $\bar{H}$. We will need the following criterion.

Lemma 5.6. With the previous notation suppose that for every $b \in \bar{F}^{\prime} \cap \bar{H}, \alpha(b)$ and $\beta(b)$ have the same orders. Then $f_{(F, H, \alpha)}=f_{(F, H, \beta)}$.

Proof. Let $\gamma$ be a linear character of $\bar{H}$ which is trivial on $\bar{F}^{\prime} \cap H$. Then there is a linear character $\tau$ of $F$ such that $\tau_{H}=\gamma$. Hence $f_{(F, H, \alpha)}=f_{(F, H, \alpha \gamma)}$. 
The conditions of the lemma imply that $\alpha_{\bar{H} \cap \bar{F}^{\prime}}$ and $\beta_{\bar{H} \cap \bar{F}^{\prime}}$ are conjugate by an element $\sigma$ of the Galois group of $\mathbb{Q}$. Hence $\alpha=\beta^{\sigma} \gamma$, where $\gamma$ is a linear character of $\bar{H}$ which is trivial on $\bar{H} \cap \bar{F}^{\prime}$. By the previous paragraph, $f_{(F, H, \alpha)}=f_{\left(F, H, \beta^{\sigma}\right)}$. On the other hand, $f_{(F, H, \beta)}=f_{\left(F, H, \beta^{\sigma}\right)}$ because there is a natural bijection between characters over $\beta$ and $\beta^{\sigma}$ conserving degrees.

Now let $V_{Q}=\left\{s_{i}=s_{i}\left(x_{q} \mid q \in Q\right)\right\} \subset F^{\prime}$ be a finite subset of $F^{\prime}$ such that $\bar{H} \cap \bar{F}^{\prime}=\left\{s_{i}[H, F]\right\}$. Suppose that $1 \rightarrow N \rightarrow G \stackrel{\phi}{\rightarrow} Q \rightarrow 1$ is an exact sequence, i.e., $\phi$ is a surjective homomorphism with kernel $N$. Let $\left\{t_{q} \mid q \in Q\right\}$ be a transversal for $N$ in $G$ such that $\phi\left(t_{q}\right)=q$, and let $\alpha$ be a $G$-invariant linear character of $N$. Define a vector

$$
V_{Q}(G, N, \phi, \alpha)=\left(o\left(\alpha\left(s_{1}\left(t_{q} \mid q \in Q\right)\right)\right), \ldots, o\left(\alpha\left(s_{k}\left(t_{q} \mid q \in Q\right)\right)\right)\right),
$$

where $k=\left|V_{Q}\right|$ and $o(r)$ denotes the order of $r$.

Corollary 5.7. (1) The vector $V_{Q}(G, N, \phi, \alpha)$ does not depend on the choice of transversal for $N$ in $G$.

(2) There exists only a finite number of possibilities for $V_{Q}(G, N, \phi, \alpha)$.

(3) Let $1 \rightarrow N_{1} \rightarrow G_{1} \stackrel{\phi_{1}}{\rightarrow} Q \rightarrow 1$ and $1 \rightarrow N_{2} \rightarrow G_{2} \stackrel{\phi_{2}}{\rightarrow} Q \rightarrow 1$ be two exact sequences and $\alpha_{i}$ a $G_{i}$-invariant linear character of $N_{i}$ for $i=1,2$. Suppose $V_{Q}\left(G_{1}, N_{1}, \phi_{1}, \alpha_{1}\right)=V_{Q}\left(G_{2}, N_{2}, \phi_{2}, \alpha_{2}\right)$. Then $f_{\left(G_{1}, N_{1}, \alpha_{1}\right)}=f_{\left(G_{2}, N_{2}, \alpha_{2}\right)}$.

Proof. (1) This is obvious because $\alpha$ is trivial on $[N, G]$.

(2) For any $b \in \bar{N} \cap \bar{G}^{\prime}, o(\alpha(b)) \leq\left|\bar{H} \cap \bar{F}^{\prime}\right|$.

(3) This is the consequence of the previous lemma.

\section{The General CASE}

In this section we finish the proof of Theorem 1.1. Let $G$ be a $p$-adic analytic group with $p>2$. We can find an open normal 2 -uniform $\operatorname{subgroup~} N$ of $G$. Since $G$ acts on N, $G$ also acts on N*. We will use the notation of Section 4 . Let $\left\{e_{1}, \cdots, e_{n}\right\}$ be a basis of $\mathrm{N}$ and $\left\{f_{1}, \cdots, f_{n}\right\}$ a basis of $\mathrm{N}^{*}$. Thus, any element $a$ from $\mathbf{N}$ or $\mathbf{N}^{*}$ is identified with a vector $\left(a_{1}, \cdots, a_{n}\right)$ from $\mathbb{Z}_{p}^{n}$.

For any subgroup $N \leq K \leq G$, define the set

$$
\operatorname{Irr}(\mathbf{N})_{K}=\left\{w \in \operatorname{Irr}(\mathbf{N}) \mid N \operatorname{St}_{G}(w)=K\right\} .
$$

Lemma 6.1. $W_{K}=\left\{(a, z) \in W \mid \pi_{z}(a) \in \operatorname{Irr}(\mathrm{N})_{K}\right\}$ is a definable set.

Proof. Fix a right transversal $\left(t_{i} \mid i=1, \ldots, m\right)$ for $N$ in $G$, and suppose that $K=$ $\bigcup_{i=1}^{s} t_{i} N$.

Note that $g_{i}(a)=a^{t_{i}}, a \in \mathbf{N}^{*}$, is a linear function of $a$ and $f(a, g)=a^{g}$, $a \in \mathrm{N}^{*}, g \in \mathrm{N}$, is an analytic function of $(a, g)$. Define a formula $F_{i}$ in the language $\mathrm{L}_{\mathrm{D}}^{\mathrm{an}}$ as

$$
F_{i}:=\exists g g_{i}(a) \equiv f(a, g)(\bmod z) .
$$

Then $W_{K}$ is equal to the definable set

$$
\left\{(a, z) \in W \mid F_{1}(a, z) \& \cdots \& F_{s}(a, z) \& \neg F_{s+1}(a, z) \& \cdots \& \neg F_{m}(z, a) \text { is true in } \mathbb{Z}_{p}\right\} \text {. }
$$


Now, fix a subgroup $K$, satisfying $N \leq K \leq G$, and put $Q=K / N$. Fix a right transversal $\left(y_{q} \mid q \in Q\right)$ for $N$ in $K$, such that $q=y_{q} N$. Let $V_{Q}=\left\{s_{j}\right\}$ be as in the previous section. For any $w \in \operatorname{Irr}(\mathrm{N})_{K}$ define $V(w)$ as follows.

Since $w \in \operatorname{Irr}(\mathbf{N})_{K}$, we have $\operatorname{St}_{G}(w) N=K$. Define $\phi: \operatorname{St}_{G}(w) \rightarrow Q$ by means of $\phi(s)=q$ if $s N=y_{q} N$, for $s \in \operatorname{St}_{G}(w)$. Then $1 \rightarrow \operatorname{St}_{N}(w) \rightarrow \operatorname{St}_{G}(w) \stackrel{\phi}{\rightarrow} Q \rightarrow 1$ is an exact sequence. Put

$$
V(w)=V_{Q}\left(\operatorname{St}_{G}(w), \operatorname{St}_{N}(w), \phi, w_{\mathrm{St}_{N}(w)}\right) .
$$

From Corollary 5.7 we know that $V(w)$ takes a finite number of values and depends only on $w$. Let $v=\left(v_{1}, \ldots, v_{k}\right)$ be such a value. We put

$$
\operatorname{Irr}(\mathrm{N})_{K, v}=\left\{w \in \operatorname{Irr}(\mathrm{N})_{K} \mid V(w)=v\right\} .
$$

Lemma 6.2. The set $W_{K, v}=\left\{(a, z) \in W_{K} \mid \pi_{z}(a) \in \operatorname{Irr}(\mathrm{N})_{K, v}\right\}$ is definable.

Proof. Note that $a\left(s_{i}\left(y_{q} a_{q} \mid q \in Q\right)\right)$ is an analytic map from $\mathrm{N}^{*} \times \mathrm{N}^{|Q|}$ to $\mathbb{Z}_{p}$ and for any $q \in Q$, the function $g_{q}(a)=a^{y_{q}}, a \in \mathrm{N}^{*}$, is a linear function of $a$. Also the function $f(a, g)=a^{g}, a \in \mathrm{N}^{*}, g \in \mathrm{N}$, is an analytic function of $(a, g)$. Define the following formula $G_{K, v}$ in $\mathrm{L}_{\mathrm{D}}^{\mathrm{an}}$ :

$$
\begin{aligned}
G_{K, v}:= & \exists\left(n_{q} \mid q \in Q\right) \forall q \in Q g_{q}(a) \equiv f\left(a,-n_{q}\right)(\bmod z) \\
& \& \forall j\left(\left(a\left(s_{j}\left(y_{q} n_{q}\right)\right) \equiv 0(\bmod z) \& v_{j}=1\right)\right. \\
& \left.\vee\left(v_{j} a\left(s_{j}\left(y_{q} n_{q}\right)\right) \equiv 0(\bmod z) \& v_{j} a\left(s_{j}\left(y_{q} n_{q}\right)\right) \not \equiv 0(\bmod p z)\right)\right) .
\end{aligned}
$$

In the first row of the formula we find a transversal $\left(y_{q} n_{q} \mid q \in Q\right)$ for $\operatorname{St}_{N}\left(\pi_{z}(a)\right)$ in $\operatorname{St}_{G}\left(\pi_{z}(a)\right)$, and in the second and third we check the condition $V\left(\pi_{z}(a)\right)=v$. Hence we have $W_{K, v}=\left\{(a, z) \in W_{K} \mid G_{K, v}(a, z)\right.$ is true in $\left.\mathbb{Z}_{p}\right\}$, and so $W_{K, v}$ is definable.

Now we are ready to prove Theorem 1.1 .

Proof of Theorem 1.1. We keep the previous notation. For any $N \leq K \leq G$ we define

$$
\zeta^{G, K}(s)=\sum_{\lambda \in \operatorname{Irr}(G \mid \chi),} \sum_{\chi \in \operatorname{Irr}(N), \operatorname{St}_{G}(\chi)=K}|\lambda(1)|^{-s} .
$$

Note that if $\lambda \in \operatorname{Irr}(G \mid \chi), \chi \in \operatorname{Irr}(N)$, and $\operatorname{St}_{G}(\chi)=K$, then $\lambda$ lies over $|G / K|$ irreducible characters of $N$. Hence we have

$$
\zeta^{G}(s)=\sum_{N \leq K \leq G} \frac{1}{|G: K|} \zeta^{G, K}(s) .
$$

So, we need to prove the rationality of $\zeta^{G, K}(s)$ for each $K$. Note that

$$
\zeta^{G, K}(s)=|G: K|^{-s} \sum_{\chi \in \operatorname{Irr}(N), \operatorname{St}_{G}(\chi)=K} f_{(K, N, \chi)} \chi(1)^{-s} .
$$

Consider an arbitrary character $\chi \in \operatorname{Irr}(N)$ such that $\operatorname{St}_{G}(\chi)=K$. Then, from Theorem 2.9, it follows that there exists a $G$-orbit $\Omega$ in $\operatorname{Irr}(\mathrm{N})$, such that $\chi=\Phi_{\Omega}$. Let $w \in \Omega$. Since $\operatorname{St}_{G}(\chi)=K$, we have $w \in \operatorname{Irr}(\mathbf{N})_{K}$. By Theorem 5.5. $f_{(K, N, \chi)}=f_{\left(\mathrm{St}_{G}(w), \mathrm{St}_{N}(w), w_{\mathrm{St}_{N}(w)}\right)}$. Then equality (6.1) can be rewritten as

$$
\zeta^{G, K}(s)=|G: K|^{-s} \sum_{w \in \operatorname{Irr}(\mathrm{N})_{K}} f_{\left(\operatorname{St}_{G}(w), \operatorname{St}_{N}(w), w_{\mathrm{St}_{N}(w)}\right)}|\mathrm{N}: \operatorname{Rad}(w)|^{-(s-2) / 2} .
$$


Now, note that if $w_{1}, w_{2} \in \operatorname{Irr}(\mathrm{N})_{K, v}$, then, by Corollary 5.7 .

$$
f_{\left(\mathrm{St}_{G}\left(w_{1}\right), \mathrm{St}_{N}\left(w_{1}\right),\left(w_{1}\right)_{\mathrm{St}_{N}\left(w_{1}\right)}\right)}=f_{\left(\operatorname{St}_{G}\left(w_{2}\right), \mathrm{St}_{N}\left(w_{2}\right),\left(w_{2}\right)_{\mathrm{St}_{N}\left(w_{2}\right)}\right)} .
$$

Hence in order to prove the rationality of $\zeta^{G, K}(s)$, it is enough to prove that $\sum_{w \in \operatorname{Irr}(\mathrm{N})_{K, v}}|\mathrm{~N}: \operatorname{Rad}(w)|^{-s}$ is a rational function in $p^{-2 s}$.

We do it in the same way that we proved Theorem 4.9, because we have that

$$
\sum_{1_{\mathrm{N}} \neq w \in \operatorname{Irr}(\mathrm{N})_{K, v}}|\mathrm{~N}: \operatorname{Rad}(w)|^{-s}=p(p-1)^{-1} \int_{W_{K, v}}|z|_{p}^{-(n+1)}|\alpha(a, z)|_{p}^{s} d a d z .
$$

We can transform the last integral to a definable integral using the argument of the proof of Theorem 4.9, because, by Lemma 6.2, $W_{K, v}$ is a definable set.

\section{An EXAmple}

Let $R$ be a complete discrete valuation ring and $\mathfrak{m}$ its maximal ideal. We suppose that the residue field $R / \mathfrak{m} \cong \mathbb{F}_{q}$ has odd characteristic. We denote by $\pi$ a generator of $\mathfrak{m}$. Put $G=\mathbb{S L}_{2}(R)$. In this section we calculate the zeta function of representations of $G$. Let $G_{i}$ be the kernel of the natural homomorphism $\mathbb{S L}_{2}(R) \rightarrow \mathbb{S L}_{2}\left(R / \mathfrak{m}^{i}\right)$. Then $G_{1}$ is an example of an $R$-perfect group (see [21] for a general definition). We will use the following facts about $G_{1}$ :

Lemma 7.1. The following properties of $G_{i}$ hold:

(1) $\left[G_{n}, G_{m}\right]_{G}=G_{n+m}$.

(2) If $n<m \leq 2 n$, then $G_{n} / G_{m}$ is an abelian group and it is isomorphic as a $G$-module to $\operatorname{sl}_{2}\left(R / \mathfrak{m}^{m-n}\right)$. Moreover, there are $G$-isomorphisms

$$
\phi_{n}: G_{n} / G_{n+1} \rightarrow \operatorname{sl}_{2}\left(\mathbb{F}_{q}\right)
$$

such that

$$
\phi_{n+m}\left(\left[x G_{n+1}, y G_{m+1}\right]_{G}\right)=\left[\phi_{n}\left(x G_{n+1}\right), \phi_{m}\left(y G_{m+1}\right)\right]_{L}
$$

for every $x \in G_{n}$ and $y \in G_{m}$.

(3) If $n<m \leq 2 n, \operatorname{Irr}\left(G_{n} / G_{m}\right)$ and $G_{n} / G_{m}$ are isomorphic as $G$-modules.

Proof. The first and second statements are well-known facts about $G_{1}$. The third statement can be obtained using the Killing form on $\operatorname{sl}_{2}\left(R / \mathfrak{m}^{m-n}\right)$.

We will consider the set $\mathbb{M}_{2}(R)$ of all 2-by-2 matrices as a Lie ring. If $x \in \mathbb{M}_{2}(R)$, then $x_{k}$ is the image of $x$ in $\mathbb{M}_{2}\left(R / \mathfrak{m}^{k}\right)$. Thus,

$$
C_{\mathbb{M}_{2}(R)}\left(x_{k}\right)=\left\{a \in \mathbb{M}_{2}(R) \mid x a-a x \in \mathfrak{m}^{k} \mathbb{M}_{2}(R)\right\} .
$$

The next lemma is the crucial point which makes possible the calculation of $\zeta^{\mathbb{S L}_{2}(R)}$.

Lemma 7.2. Let $x \in \mathrm{sl}_{2}(R) \backslash \mathfrak{m} \mathrm{sl}_{2}(R)$ and $k \geq 1$. Then

$$
C_{\mathbb{M}_{2}(R)}\left(x_{k}\right)=C_{\mathbb{M}_{2}(R)}(x)+\mathfrak{m}^{k} \mathbb{M}_{2}(R)=R+R x+\mathfrak{m}^{k} \mathbb{M}_{2}(R) .
$$

In particular, $C_{G}\left(x_{k}\right)=C_{G}(x) G_{k}$.

Proof. The inclusions $C_{\mathbb{M}_{2}(R)}\left(x_{k}\right) \geq C_{\mathbb{M}_{2}(R)}(x)+\mathfrak{m}^{k} \mathbb{M}_{2}(R) \geq R+R x+\mathfrak{m}^{k} \mathbb{M}_{2}(R)$ are evident. Let us prove that $C_{\mathbb{M}_{2}(R)}\left(x_{k}\right) \leq R+R x+\mathfrak{m}^{k} \mathbb{M}_{2}(R)$. We will prove it by induction on $k$. Note that $C_{\mathbb{M}_{2}\left(\mathbb{F}_{q}\right)}\left(x_{1}\right)=\mathbb{F}_{q}+\mathbb{F}_{q} x_{1}$, because $x_{1}$ is a noncentral element of $\mathbb{M}_{2}\left(\mathbb{F}_{q}\right)$. Thus,

$$
C_{\mathbb{M}_{2}(R)}\left(x_{k}\right) \leq C_{\mathbb{M}_{2}(R)}\left(x_{1}\right)=R+R x+\mathfrak{m} \mathbb{M}_{2}(R) .
$$


In particular, the lemma holds when $k=1$. Now, suppose that $k \geq 2$ and the lemma holds for $k-1$. Since $C_{\mathbb{M}_{2}(R)}\left(x_{k}\right) \cap \mathfrak{m} \mathbb{M}_{2}(R)=\mathfrak{m} C_{\mathbb{M}_{2}(R)}\left(x_{k-1}\right)$, we obtain from the inductive hypothesis and (7.1) that

$$
\begin{aligned}
C_{\mathbb{M}_{2}(R)}\left(x_{k}\right) & \leq R+R x+\left(C_{\mathbb{M}_{2}(R)}\left(x_{k}\right) \cap \mathfrak{m M}_{2}(R)\right) \\
& =R+R x+\mathfrak{m} C_{\mathbb{M}_{2}(R)}\left(x_{k-1}\right)=R+R x+\mathfrak{m}^{k} \mathbb{M}_{2}(R) .
\end{aligned}
$$

Lemma 7.3. Let $m \geq 1$ and $\tau \in \operatorname{Irr}\left(G_{m} / G_{2 m+1} \mid G_{2 m} / G_{2 m+1}\right)$. Then $\tau(1)=q$.

Proof. The group $H=G_{m} / G_{2 m+1}$ has nilpotency class 2. Hence,

$$
\tau(1)=|H: Z(\tau)|^{1 / 2},
$$

where $Z(\tau)$ is the center of $\tau$. Let $v$ be the irreducible constituent of $\tau_{G_{2 m} / G_{2 m+1}}$ and $\alpha$ the bilinear form on $G_{m} / G_{m+1}$ defined by $\alpha\left(x G_{m}, y G_{m}\right)=v\left([x, y]_{G}\right)$. Then from Lemma 7.1(2) we obtain that since $v$ is not trivial, the radical of $\alpha$ has size $q$. But the preimage of the radical of $\alpha$ in $G_{m}$ is exactly $Z(\tau)$. Thus $\tau(1)=q$.

Let $\lambda \in \operatorname{Irr}(G)$. We define the level $n(\lambda)$ of $\lambda$ to be the least number $n \geq 0$ such that $G_{n+1} \leq \operatorname{ker} \lambda$. The characters of $G$ of level 0 are well known: they are characters of the group $\mathbb{S L}_{2}\left(\mathbb{F}_{q}\right)$. We fix our attention on characters of positive level. We divide these characters into three groups as follows. Let $\lambda \in \operatorname{Irr}(G)$ be of level $n>0$ and $v_{\lambda}$ an irreducible constituent of $\lambda_{G_{n}}$. Since $v_{\lambda}$ is an irreducible character of $G_{n} / G_{n+1}$, using Lemma 7.1 we can consider $v_{\lambda}$ as an element of $\operatorname{sl}_{2}\left(\mathbb{F}_{q}\right)$. Then we define the type of $\lambda$ as follows:

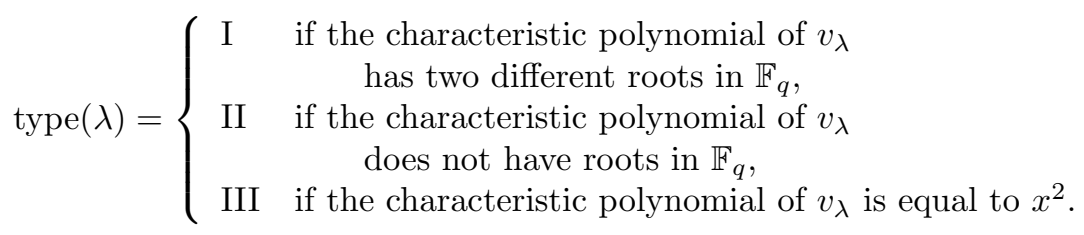

Although the choice of $v_{\lambda}$ is not unique, the definition of the type of $\lambda$ only depends on $\lambda$.

Theorem 7.4. Let $\lambda$ be an irreducible character of $G$ of level $n>0$. Then

$$
\lambda(1)= \begin{cases}(q+1) q^{n} & \text { if type }(\lambda)=I, \\ (q-1) q^{n} & \text { if type }(\lambda)=I I, \\ \frac{q^{2}-1}{2} q^{n-1} & \text { if type }(\lambda)=I I I .\end{cases}
$$

Proof. Put $m=\lfloor n / 2\rfloor+1$, and let $\chi$ be an irreducible constituent of $\lambda_{G_{m}}$. By Lemma 7.1. there exists an isomorphism $\phi: \operatorname{Irr}\left(G_{m} / G_{n+1}\right) \cong \operatorname{sl}_{2}\left(R / \mathfrak{m}^{n-m+1}\right)$ of $G$-modules. Hence there exists $x \in \operatorname{sl}_{2}(R) \backslash \mathfrak{m s l}_{2}(R)$ such that $\phi(\chi)=x_{n-m+1}$. Without loss of generality we can assume that $v_{\lambda}=\chi_{G_{n}}$. Then we have that $\operatorname{St}_{G}\left(v_{\lambda}\right)=C_{G}\left(x_{1}\right)$.

First suppose that $n$ is an odd number. By Lemma 7.2

$$
\operatorname{St}_{G}(\chi)=C_{G}\left(x_{n-m+1}\right)=A G_{m},
$$

where $A=C_{G}(x)$ is an abelian subgroup of $G$. Thus, we can extend $\chi$ to $\operatorname{St}_{G}(\chi)$ and so $\lambda(1)=\left|G: \operatorname{St}_{G}(\chi)\right|$. 
Now suppose that $n$ is an even number. Again, we have that $\operatorname{St}_{G}(\chi)=A G_{m-1}$ for some abelian subgroup $A$ of $G$. Let $\tau$ be an irreducible constituent of $\lambda_{G_{m-1}}$ lying over $\chi$.

First we will show that $\operatorname{St}_{G}(\tau)=\operatorname{St}_{G}(\chi)$. The inclusion $\operatorname{St}_{G}(\tau) \leq \operatorname{St}_{G}(\chi)$ is clear. In order to prove the inverse inclusion we have to show that $A$ stabilizes $\tau_{Z}$ where $Z=Z(\tau)$ is the center of $\tau$, because $\tau$ vanishes outside $Z$. Note that

$$
Z=G \cap\left\{1+\pi^{m-1} t \mid t \in C_{\mathbb{M}_{2}(R)}\left(x_{1}\right)\right\} .
$$

Thus, by Lemma 7.2 . $[Z, A]_{G}=\left[G_{m}, A\right]_{G}$ and $G_{m-1} \cap A \leq Z$. In particular, $A$ fixes $\tau_{Z}$ and so $A$ fixes $\tau$.

Now we will prove that we can extend $\tau$ to $A G_{m-1}$. Let $P$ be the pro- $p$ Sylow subgroup of $A$. We only need to show that $\tau$ can be extended to $P G_{m-1}$. The idea of the following argument is taken from the proof of Theorem 5.5. As we see, it also works when $R$ has positive characteristic. Define a new operation on $G_{m-1} / G_{n+1}$ :

$$
x * y=x y[x, y]^{1 / 2} .
$$

We obtain that $G_{m-1} / G_{n+1}$ is a $G$-module with respect to this operation. Moreover, $M=\left(G_{m-1} / G_{n+1}, *\right)$ is isomorphic to $\operatorname{sl}_{2}\left(R / \mathfrak{m}^{n-m+2}\right)$ as a $G$-module. Indeed, it is easy to check that

$$
1+a \quad\left(\bmod \mathfrak{m}^{n+1} \mathbb{M}_{2}(R)\right) \mapsto a-\frac{a^{2}}{2} \quad\left(\bmod \mathfrak{m}^{n-m+2} \mathbb{M}_{2}(R)\right)
$$

is a $G$-isomorphism between

$$
M \text { and }\left(\mathfrak{m}^{m-1} \operatorname{sl}_{2}(R)+\mathfrak{m}^{n-m+2} \mathbb{M}_{2}(R)\right) / \mathfrak{m}^{n-m+2} \mathbb{M}_{2}(R) .
$$

Recall that $A=C_{G}(x)$. We can find an extension $\mu \in \operatorname{Irr}(M)$ of $\chi$ such that $A$ fixes $\mu$. As in the proof of Lemma 7.3, we define a bilinear form $\alpha$ on $G_{m-1} / G_{m}$ by means of $\alpha\left(x G_{m}, y G_{m}\right)=\mu\left([x, y]_{G}\right)=v_{\lambda}\left([x, y]_{G}\right), x, y \in G_{m-1}$. By Lemma 2.4. there exists $G_{m} \leq D \leq G_{m-1}$ such that $D / G_{m}$ is a maximal isotropic subspace of $G_{m-1} / G_{m}$ and $P$ fixes $D$. It is clear that $D$ is a subgroup of $G_{m-1}$ and $\mu_{D}$ is a linear character of $D$. Note that $\left|G_{m-1}: D\right|=q$, whence $\tau=\mu^{G_{m-1}}$. Since $P$ is abelian and $P$ fixes $\mu_{D}, \mu_{D}$ can be extended to $P D$. Hence, by Corollary 5.3, $\tau$ can be extended to $P G_{m-1}$ and so to $A G_{m-1}$. Therefore, $\lambda(1)=\left|G: \operatorname{St}_{G}(\chi)\right| q$.

Thus, in both cases when $n$ is odd and when $n$ is even we reduce the calculation of $\lambda(1)$ to the calculation of $\left|G: \operatorname{St}_{G}(\chi)\right|=\left|G: C_{G}\left(x_{n-m+1}\right)\right|$. Applying Lemma 7.2, we obtain that

$$
\left|G: C_{G}\left(x_{k+1}\right)\right|= \begin{cases}(q+1) q^{2 k+1} & \text { if type }(\lambda)=\mathrm{I} \\ (q-1) q^{2 k+1} & \text { if type }(\lambda)=\mathrm{II} \\ \frac{q^{2}-1}{2} q^{2 k} & \text { if type }(\lambda)=\mathrm{III}\end{cases}
$$

This gives the theorem.

Theorem 7.5. Let $R$ be as before. Then

$$
\begin{aligned}
\zeta^{S_{2}(R)} & =1+q^{-s}+\frac{q-3}{2}(q+1)^{-s}+2\left(\frac{q+1}{2}\right)^{-s}+\frac{q-1}{2}(q-1)^{-s}+2\left(\frac{q-1}{2}\right)^{-s} \\
& +\frac{4 q\left(\frac{q^{2}-1}{2}\right)^{-s}+\frac{q^{2}-1}{2}\left(q^{2}-q\right)^{-s}+\frac{(q-1)^{2}}{2}\left(q^{2}+q\right)^{-s}}{1-q^{-s+1}}
\end{aligned}
$$


Proof. From [9, page 155] we know that when $q$ is odd,

$\zeta^{\mathbb{S L}_{2}\left(\mathbb{F}_{q}\right)}=1+q^{-s}+\frac{q-3}{2}(q+1)^{-s}+2\left(\frac{q+1}{2}\right)^{-s}+\frac{q-1}{2}(q-1)^{-s}+2\left(\frac{q-1}{2}\right)^{-s}$.

The characters of $\mathbb{S L}_{2}\left(\mathbb{F}_{q}\right)$ are exactly the characters of $\mathbb{S L}_{2}(R)$ of level 0. Let $n>0$ and $v \in \operatorname{Irr}\left(G_{n} / G_{n+1}\right)$. Then $|\operatorname{Irr}(G \mid v)|=\left|\operatorname{Irr}\left(\operatorname{St}_{G}(v) \mid v\right)\right|$. Since all characters from $\operatorname{Irr}(G \mid v)$ have the same type and the same level, the previous theorem implies that they have the same degree. Therefore, since

we obtain that

$$
\sum_{\theta \in \operatorname{Irr}\left(\operatorname{St}_{G}(v) \mid v\right)} \theta(1)^{2}=\left|\operatorname{St}_{G}(v): G_{n}\right|,
$$

$$
|\operatorname{Irr}(G \mid v)|=\frac{\left|G: \operatorname{St}_{G}(v)\right|^{2}\left|\operatorname{St}_{G}(v): G_{n}\right|}{\lambda(1)^{2}}=\frac{\left|G: \operatorname{St}_{G}(v)\right|\left|G: G_{n}\right|}{\lambda(1)^{2}},
$$

where $\lambda$ is any character from $\operatorname{Irr}(G \mid v)$.

For example, we calculate the number of the irreducible characters of level $n$ and type I. In $\mathrm{sl}_{2}\left(\mathbb{F}_{q}\right)$ there are $(q-1) / 2 G$-conjugacy classes of diagonalizable nontrivial elements. Hence, by (7.2) and Theorem [7.4, the number of irreducible characters of level $n$ and type $\mathrm{I}$ is equal to

$$
\frac{q-1}{2} \frac{q(q+1) q^{3 n-2}(q-1)(q+1)}{(q+1)^{2} q^{2 n}}=\frac{q^{n-1}(q-1)^{2}}{2} .
$$

The number of irreducible characters of level $n$ and type II is equal to

$$
\frac{q-1}{2} \frac{q(q-1) q^{3 n-2}(q-1)(q+1)}{(q-1)^{2} q^{2 n}}=\frac{q^{n-1}\left(q^{2}-1\right)}{2} .
$$

The number of irreducible characters of level $n$ and type III is equal to

$$
2 \frac{\frac{(q+1)(q-1)}{2} q^{3 n-2}(q-1)(q+1)}{\frac{\left(q^{2}-1\right)^{2}}{4} q^{2 n-2}}=4 q^{n} .
$$

Applying Theorem 7.4, we obtain the desired formula for $\zeta^{\mathrm{SL}_{2}(R)}$.

\section{ACKNOWLEDGMENT}

The author would like to thank Benjamin Martin for some helpful comments on a preliminary version of this work.

\section{REFERENCES}

1. H. Bass, A. Lubotzky, A. R. Magid, S. Mozes, The Proalgebraic Completion of Rigid Groups, Geom. Dedicata 95 (2002), 19-58. MR.1950883 (2004c:20069)

2. N. Bourbaki, Lie groups and Lie algebras, Springer-Verlag, 1989. MR0979493 (89k:17001)

3. J. Denef, The rationality of the Poincaré series associated to the p-adic points on a variety, Invent. Math. 77 (1984), 1-23. MR0751129 (86c:11043)

4. J. Denef, Multiplicity of the poles of the Poincare series of a p-adic subanalytic set, Seminaire de Theorie des Nombres de Bordeaux (1987-1988), exposé N43, 1-8. MR0993136 (90f:11089)

5. J. Denef, Arithmetic and geometric applications of quantifier elimination for valued fields, Model theory, algebra, and geometry, pp. 173-198, Math. Sci. Res. Inst. Publ., 39, Cambridge Univ. Press, Cambridge, 2000. MR.1773707 (2001e:03063)

6. J. Denef, L. van den Dries, p-adic and real subanalytic sets, Ann. of Math. (2) 128 (1988), 79-138. MR0951508 (89k:03034)

7. J. Dixon, M. du Sautoy, A. Mann, D. Segal, Analytic pro-p groups, 2nd ed., Cambridge University Press, Cambridge, 1999. MR 1720368 (2000m:20039) 
8. M. du Sautoy, Finitely generated groups, p-adic analytic groups and Poincaré series, Ann. of Math. (2) 137 (1993), 639-670. MR.1217350 (94j:20029)

9. F. Digne, J. Michel, Representations of Finite Groups of Lie Type, Cambridge University Press, Cambridge, 1991. MR:1118841 (92g:20063)

10. J. Gonzalez-Sanchez, A. Jaikin-Zapirain, On the structure of normal subgroups of potent pgroups, J. of Algebra 276 (2004), 193-209. MR2054394 (2005d:20034)

11. R. Howe, On representations of discrete, finitely generated, torsion-free, nilpotent groups, Pacific J. Math. 73 (1977), 281-305. MR0499004 (58:16984)

12. R. Howe, Kirillov theory for compact p-adic groups, Pacific J. Math. 73 (1977), 365-381. MR 0579176 (58:28314)

13. I. Ilani, Analytic pro-p groups and their Lie algebras, J. Algebra 176 (1995), 34-58. MR 1345293 (96h:20059)

14. I. M. Isaacs, Character theory of finite groups, Dover Publ., New York, 1976. MR0460423 $(57: 417)$

15. I. M. Isaacs, Characters of $\pi$-separable groups, J. Algebra 86 (1984), 98-128. MR0727371 (85h:20012)

16. A. A. Kirillov, Merits and demerits of the orbit method, Bull. Amer. Math. Soc. 36 (1999), 433-488. MR.1701415 (2000h:22001)

17. B. Klopsch, On the Lie theory of p-adic analytic groups, Math. Z. 249 (2005), 713-730. MR 2126210

18. E. I. Khukhro, $p$-Automorphisms of Finite p-Groups, Cambridge University Press, Cambridge, 1998. MR1615819 (99d:20029)

19. M. Lazard, Groupes analytiques p-adiques, Publ. Math. I.H.E.S. 26 (1965), 389-603. MR.0209286 (35:188)

20. A. Lubotzky, B. Martin, Polynomial Representation Growth and the Congruence Subgroup Problem, Israel J. Math. 144 (2004), 293-316. MR2121543

21. A. Lubotzky, A. Shalev, On some $\Lambda$-analytic pro-p groups, Israel J. Math. 85 (1994), 307-337. MR.1264349 (95f:20047)

22. C. C. Macduffee, The theory of matrices, Chelsea Publishing Company, New York.

23. M. Suzuki, Group Theory I, Springer-Verlag, 1982. MR0648772 (82k:20001c)

Departamento de Matemáticas, Facultad de Ciencias, Universidad Autónoma de MADRID, 28049 MADRID, SPAIN

E-mail address: andrei.jaikin@uam.es 